\title{
STABILIZATION METHODS IN RELAXED MICROMAGNETISM
}

\author{
Stefan A. Funken ${ }^{1}$ And Andreas Prohl ${ }^{2}$
}

\begin{abstract}
The magnetization of a ferromagnetic sample solves a non-convex variational problem, where its relaxation by convexifying the energy density resolves relevant macroscopic information. The numerical analysis of the relaxed model has to deal with a constrained convex but degenerated, nonlocal energy functional in mixed formulation for magnetic potential $u$ and magnetization $\mathbf{m}$. In [C. Carstensen and A. Prohl, Numer. Math. 90 (2001) 65-99], the conforming $P 1-(P 0)^{d}$-element in $d=2,3$ spatial dimensions is shown to lead to an ill-posed discrete problem in relaxed micromagnetism, and suboptimal convergence. This observation motivated a non-conforming finite element method which leads to a well-posed discrete problem, with solutions converging at optimal rate. In this work, we provide both an a priori and a posteriori error analysis for two stabilized conforming methods which account for inter-element jumps of the piecewise constant magnetization. Both methods converge at optimal rate; the new approach is applied to a macroscopic nonstationary ferromagnetic model [M. Kružík and A. Prohl, Adv. Math. Sci. Appl. 14 (2004) 665-681-M. Kružík and T. Roubíček, Z. Angew. Math. Phys. 55 (2004) 159-182].
\end{abstract}

Mathematics Subject Classification. 65K10, 65N15, 65N30, 65N50, 73C50, 73S10.

Received: January 28, 2005. Revised: May 4, 2005.

\section{INTRODUCTION}

The mathematical description of magnetization states $\mathbf{m}: \omega \rightarrow \mathcal{S}^{d-1}:=\left\{\mathbf{x} \in \mathbb{R}^{d}|| \mathbf{x} \mid=1\right\} \subset \mathbb{R}^{d}$ in rigid ferromagnetic bodies that cover a domain $\omega \subset \mathbb{R}^{d}, d=2,3$ relies on the classical model by Weiss et al. [2], where the magnetization minimizes the magnetostatic energy

$$
E(\mathbf{m})=\alpha \int_{\omega}|\nabla \mathbf{m}|^{2} \mathrm{~d} \mathbf{x}+\int_{\omega} \phi(\mathbf{m}) \mathrm{d} \mathbf{x}+\frac{1}{2} \int_{\Omega}|\nabla u|^{2} \mathrm{~d} \mathbf{x}-\int_{\omega}\langle\mathbf{f}, \mathbf{m}\rangle \mathrm{d} \mathbf{x}
$$

subject to the following consequence of Maxwell's equations,

$$
-\Delta u+\operatorname{div}\left(\chi_{\omega} \mathbf{m}\right)=0 \quad \text { in } H^{-1}(\Omega) .
$$

Here, $\chi_{\omega}: \Omega \rightarrow \mathbb{R}$ denotes the characteristic function of the set $\omega$, i.e., $\chi_{\omega}(\mathbf{x})=1$ if $\mathbf{x} \in \omega$, and $\chi_{\omega}(\mathbf{x})=0$ if not. Further, to account for non-local energy contributions, we choose a bounded set $\mathbb{R}^{d} \supset \supset \Omega \supset \supset \omega$ instead

\footnotetext{
Keywords and phrases. Micromagnetics, stationary, nonstationary, microstructure, relaxation, nonconvex minimization, degenerate convexity, finite elements methods, stabilization, penalization, a priori error estimates, a posteriori error estimates.

1 Department of Numerical Analysis, University of Ulm, 89069 Ulm, Germany. stefan.funken@uni-ulm.de

2 Department of Mathematics, ETHZ, 8092 Zürich, Switzerland.
} 
of $\mathbb{R}^{d}$; for a different approach that couples finite element methods and boundary element methods we refer to $[3,4]$.

The magnetic energy consists of four contributions which favour different magnetic properties: the first term ("surface energy") is scaled by $\alpha>0$ and favours non-oscillatory structures. The non-convex anisotropic energy density $0 \leq \phi(\cdot)$ reflects crystallographic properties of the ferromagnet, like uniaxial (e.g., iron) or cubic (e.g., cobalt-samarium) materials [8]. In the uniaxial case which is considered below, there holds $\phi(\mathbf{m})=0$, if $\mathbf{m}= \pm \mathbf{e}$, for a given (constant) easy axis $\mathbf{e} \in \mathcal{S}^{d-1}$. The third term in (1.1) takes into account applied exterior fields $\mathbf{f}: \omega \rightarrow \mathbb{R}^{d}$. Finally, the stray-field energy measures how much the magnetization deviates from being divergence-free (i.e., $\nabla u$ is the Helmholtz projection of $\mathbf{m}$, for $u: \Omega \rightarrow \mathbb{R}$ the magnetic potential).

As far as applications are concerned, we have mainly "macroscopic" bodies in mind which justifies deleting the first term in (1.1) from the magnetic energy $(\alpha=0)$ [6]. With this modification in (1.1), the fundamental problem in micromagnetism reads

$$
\min _{\boldsymbol{\mu} \in \mathcal{A}} E(\boldsymbol{\mu}), \quad \text { where } \mathcal{A}:=\left\{\boldsymbol{\mu} \in L^{2}(\omega)^{d},|\boldsymbol{\mu}|=1, \text { a.e. in } \omega\right\} .
$$

Note that (1.3) does not have any solution in general that can be inferred from direct minimization method since $\mathcal{A}$ is not weakly closed in $L^{2}(\omega)^{d}$, and we have to extend the notion of solution to Young measure-valued solutions. A proper relaxation is $\bar{E}: \overline{\mathcal{A}} \rightarrow \mathbb{R}[16]$, where

$$
\overline{\mathcal{A}}=\left\{\nu=\left\{\nu_{\mathbf{x}}\right\}_{\mathbf{x} \in \omega} \mid \operatorname{supp} \nu_{\mathbf{x}}=\mathcal{S}^{d-1}, \mathbf{x} \mapsto \nu_{\mathbf{x}} \text { weakly measurable }\right\} \subset L^{1}\left(\omega ; C\left(\mathcal{S}^{d-1}\right)\right)^{*} \equiv L_{\mathrm{w}}^{\infty}\left(\omega ; \mathcal{M}\left(\mathcal{S}^{d-1}\right)\right),
$$

and

$$
\bar{E}(\nu)=\int_{\omega} \int_{\mathcal{S}^{d-1}} \phi(\mathbf{A}) \nu_{\mathbf{x}}(\mathrm{d} \mathbf{A}) \mathrm{d} \mathbf{x}+\frac{1}{2} \int_{\Omega}|\nabla u|^{2} \mathrm{~d} \mathbf{x}-\int_{\omega}\langle\mathbf{f}, \mathbf{m}\rangle \mathrm{d} \mathbf{x}, \quad \text { where } \quad \mathbf{m}(\mathbf{x})=\int_{\mathcal{S}^{d-1}} \mathbf{A} \nu_{\mathbf{x}}(\mathrm{d} \mathbf{A})
$$

for $\Delta u=\operatorname{div}\left(\chi_{\omega} \mathbf{m}\right)$ in $\Omega \subseteq \mathbb{R}^{d}$, which is studied numerically in [11]. Another possibility is to convexify the energy density $\phi(\cdot)$ instead to obtain a degenerate energy functional $E^{\star \star}(\cdot)$ that attains minimizers which gather (averaged) macroscopic properties of the ferromagnet [6], with the set $\mathcal{A}$ being replaced with $\mathcal{A}^{\star \star}:=\left\{\boldsymbol{\mu} \in L^{2}(\omega)^{d},|\boldsymbol{\mu}| \leq 1\right.$, a.e. in $\left.\omega\right\}$. Since $\phi^{\star \star}(\cdot)$ is assumed to be Fréchet-differentiable, and further using the non-negative Lagrange multiplier $\lambda \in L^{2}(\omega)$ associated to the constraint $|\mathbf{m}| \leq 1$, we have to solve the corresponding Euler-Lagrange equation $(\mathbf{P})$ : Seek $(u, \mathbf{m}, \lambda) \in H_{0}^{1}(\Omega) \times L^{2}(\omega)^{d} \times L^{2}(\omega)$ such that

$$
\begin{aligned}
& \int_{\Omega}\langle\nabla u, \nabla w\rangle \mathrm{d} \mathbf{x}=\int_{\omega}\langle\mathbf{m}, \nabla w\rangle \mathrm{d} \mathbf{x} \quad \forall w \in H_{0}^{1}(\Omega), \\
& \nabla u+D \phi^{* *}(\mathbf{m})+\lambda \mathbf{m}=\mathbf{f} \quad \text { a.e. in } \omega, \\
& 0 \leq \lambda, \quad|\mathbf{m}| \leq 1, \quad \text { and } \quad \lambda(1-|\mathbf{m}|)_{+}=0 \quad \text { a.e. in } \omega .
\end{aligned}
$$

We denote $(s)_{+}:=\max \{s, 0\}$, and the last condition in (1.6) states that $\lambda \neq 0$ is possible only for $|\mathbf{m}|=1$, as a consequence from $\lambda \mathbf{m} \in \partial \psi(\mathbf{m})$ for the convex characteristic functional $\psi: \mathbb{R}^{d} \rightarrow[0, \infty]$ defined by $\psi(\mathbf{m})=0$ if $|\mathbf{m}| \leq 1$, and $\psi(\mathbf{m})=\infty$ if not.

Remark 1.1. For uniaxial magnets with easy axis $\mathbf{e} \in \mathcal{S}^{d-1}$, the Young measure generated by minimizing sequences in (1.3) can be obtained from solutions to (1.4)-(1.6) by the following formula $(c f$. [6]),

$$
\begin{gathered}
\nu_{\mathbf{x}}=\lambda(\mathbf{m}) \delta_{\mathbf{m}^{+}(\mathbf{m})}+(1-\lambda(\mathbf{m})) \delta_{\mathbf{m}^{-}(\mathbf{m})}, \\
\mathbf{m}^{ \pm}(\mathbf{m}):= \pm\left(1-\left|\left\langle\mathbf{m}, \mathbf{e}_{\perp}\right\rangle\right|^{2}\right)^{1 / 2} \mathbf{e}+\left\langle\mathbf{m}, \mathbf{e}_{\perp}\right\rangle \mathbf{e}_{\perp}, \quad \text { and } \lambda(\mathbf{m}):=\frac{1}{2}+\frac{\langle\mathbf{m}, \mathbf{e}\rangle}{2\left(1-\left\langle\mathbf{m}, \mathbf{e}_{\perp}\right\rangle^{2}\right)^{1 / 2}} .
\end{gathered}
$$

As is shown in [6], Theorem 4.2, Problem (P) has a solution. Moreover, solutions are unique in the uniaxial case, where the convexified energy density is of the form $\phi^{\star \star}(\mathbf{m})=\frac{1}{2}\left|\left\langle\mathbf{m}, \mathbf{e}_{\perp}\right\rangle\right|^{2}, c f$. [5]. A corresponding uniqueness 
result does not hold for cubic materials, in general. For this reason, the uniaxial case is a good platform for the subsequent numerical analysis of stationary ferromagnetism.

A first numerical modelling and analysis for Problem $(\mathbf{P})$ in the case $d=2$ is given in [5]; here, both theoretical as well as computational analysis are given which evidence failure of the most simple choice of finite element ansatz spaces, which are piecewise constant magnetizations $\mathbf{m}_{h}: \omega \rightarrow \mathbb{R}^{d}$ and piecewise affine, globally continuous potentials $u_{h}: \Omega \rightarrow \mathbb{R}$. Instead, a non-conforming ansatz using the Crouzeix-Raviart element is proposed to the problem and shown to give a well-posed problem (uniaxial case), leading to optimal convergence behavior of the method. Secondly, the nonlinear side constraint on the pointwise modulus of the magnetization is ensured via a penalization approach. The analysis is complemented by a posteriori error estimates which show an efficiency-reliability gap that is caused by the strongly nonlinear character of the problem and restricted regularity properties of the solution to $(\mathbf{P})$.

The main goal of the present work is to come back to the non-stable ansatz sketched before which uses conforming finite elements, together with a stabilization strategy for a penalized version of Problem $(\mathbf{P})$.

The discrete penalized, stabilized problem $\left(\mathbf{P}_{\varepsilon, N}^{\ell} \mid \mathcal{S}_{0}^{1}(\mathcal{T}) \times \mathcal{L}^{0}\left(\left.\mathcal{T}\right|_{\omega}\right)^{d} \times \mathcal{L}^{0}\left(\left.\mathcal{T}\right|_{\omega}\right)\right)$, or $\left(\mathbf{P}_{\varepsilon, N}^{\ell}\right)$, for $\ell \in\{\mathrm{A}, \mathrm{B}\}$ shortly, reads as follows, $\beta_{\mathcal{E}}>0$ : Seek $\left(u_{h}, \mathbf{m}_{h}, \lambda_{h}\right) \in \mathcal{S}_{0}^{1}(\mathcal{T}) \times \mathcal{L}^{0}\left(\left.\mathcal{T}\right|_{\omega}\right)^{d} \times \mathcal{L}^{0}\left(\left.\mathcal{T}\right|_{\omega}\right)$ such that for all $\left(w_{h}, \boldsymbol{\mu}_{h}\right) \in \mathcal{S}_{0}^{1}(\mathcal{T}) \times \mathcal{L}^{0}\left(\left.\mathcal{T}\right|_{\omega}\right)^{d}$,

$$
\begin{aligned}
& \left(\nabla u_{h}, \nabla w_{h}\right)_{\Omega}-\left(\mathbf{m}_{h}, \nabla w_{h}\right)_{\omega}=0 \\
& \left(\nabla u_{h}, \boldsymbol{\mu}_{h}\right)_{\omega}+\left(D \phi^{* *}\left(\mathbf{m}_{h}\right), \boldsymbol{\mu}_{h}\right)_{\omega}+\left(\lambda_{h} \mathbf{m}_{h}, \boldsymbol{\mu}_{h}\right)_{\omega}+t_{\ell}\left(\left[\mathbf{m}_{h}\right],\left[\boldsymbol{\mu}_{h}\right]\right)=\left(\mathbf{f}_{\mathcal{T}}, \boldsymbol{\mu}_{h}\right)_{\omega}, \\
& \lambda_{h}=\varepsilon^{-1} \frac{\left(\left|\mathbf{m}_{h}\right|-1\right)_{+}}{\left|\mathbf{m}_{h}\right|} \quad \text { a.e. in } \omega .
\end{aligned}
$$

Here, [.] denotes the jump across inter-element (interior) faces $\left.E \subset \mathcal{E}\right|_{\omega}:=\bigcup_{T \in \mathcal{T} \mid{ }_{\omega}} \partial T$, according to a given but arbitrary orientation of triangles $T \in \mathcal{T}$. Alternatively, we deal with the following stabilization ansatzes,

$$
\begin{aligned}
& t_{\mathrm{A}}\left(\left[\mathbf{m}_{h}\right],\left[\boldsymbol{\mu}_{h}\right]\right):=\sum_{\left.E \subset \mathcal{E}\right|_{\omega}} \beta_{E} h_{E} \int_{E}\left[\left\langle\mathbf{m}_{h}, \mathbf{n}\right\rangle\right]\left[\left\langle\boldsymbol{\mu}_{h}, \mathbf{n}\right\rangle\right] \mathrm{d} s, \\
& t_{\mathrm{B}}\left(\left[\mathbf{m}_{h}\right],\left[\boldsymbol{\mu}_{h}\right]\right):=\sum_{\left.E \subset \mathcal{E}\right|_{\omega}} \beta_{E} h_{E} \int_{E}\left\langle\left[\mathbf{m}_{h}\right],\left[\boldsymbol{\mu}_{h}\right]\right\rangle \mathrm{d} s,
\end{aligned}
$$

with $\left.\left(\beta_{\mathcal{E}}\right)\right|_{E}=\beta_{E}>0$. Finally, other numerical scheme fit into this framework that employ element-wise affine, globally continuous magnetizations, $\mathbf{m}_{h}, \boldsymbol{\mu}_{h} \in \mathcal{S}^{1}\left(\left.\mathcal{T}\right|_{\omega}\right)^{d}$ in (1.9)-(1.11) and replace $t_{\ell}\left(\left[\mathbf{m}_{h}\right],\left[\boldsymbol{\mu}_{h}\right]\right)$ in (1.10) with $d_{\ell}\left(\mathbf{m}_{h}, \boldsymbol{\mu}_{h}\right)$,

$$
d_{\mathrm{A}}\left(\mathbf{m}_{h}, \boldsymbol{\mu}_{h}\right):=\sum_{\left.T \in \mathcal{T}\right|_{\omega}} \beta_{T} h_{T}^{2}\left(\operatorname{div} \mathbf{m}_{h}, \operatorname{div} \boldsymbol{\mu}_{h}\right)_{T}, \quad d_{\mathrm{B}}\left(\mathbf{m}_{h}, \boldsymbol{\mu}_{h}\right):=\sum_{\left.T \in \mathcal{T}\right|_{\omega}} \beta_{T} h_{T}^{2}\left(\nabla \mathbf{m}_{h}, \nabla \boldsymbol{\mu}_{h}\right)_{T}
$$

for $\left.\left(\beta_{\mathcal{T}}\right)\right|_{T}=\beta_{T}>0$. We mention some physical relevancy of $d_{\mathrm{B}}(\cdot, \cdot)$, whereas $d_{\mathrm{A}}(\cdot, \cdot)$ is only motivated from numerical reasons.

In our analysis, we confine to the investigation of the stabilization methods $t_{\ell}, \ell \in\{\mathrm{A}, \mathrm{B}\}$ in $(1.9)-(1.11)$ that incorporate jump terms to the scheme, and which allow for piecewise constant finite element magnetizations. Apart from this reasoning, lower dimensional discrete problems (in practice) and significantly simplified a priori and a posteriori error analysis with broader range of applicability make these stabilization methods attractive. 
In the following, we suppose that $\phi^{\star \star}(\cdot)$ satisfies for all $\mathbf{m}_{1}, \mathbf{m}_{2} \in \mathbb{R}^{d}$, and $c>0$,

$$
c\left|D \phi^{\star \star}\left(\mathbf{m}_{2}\right)-D \phi^{\star \star}\left(\mathbf{m}_{1}\right)\right|^{2} \leq\left\langle D \phi^{\star \star}\left(\mathbf{m}_{2}\right)-D \phi^{\star \star}\left(\mathbf{m}_{1}\right), \mathbf{m}_{2}-\mathbf{m}_{1}\right\rangle,
$$

which covers the uniaxial case $(c=1)$, in particular.

Theorem 1.1. Let $\mathcal{T}$ be a quasiuniform triangulation of the domain $\Omega \subset \mathbb{R}^{d}, d=2,3$ that satisfies for $\left.E_{i} \in \mathcal{E}\right|_{\omega}$,

$$
\exists \boldsymbol{\xi}=\bigcap_{i=1, . ., d} E_{i} \in \omega \quad \text { such that } \int_{\omega}\left\langle\nabla \phi_{\boldsymbol{\xi}}, \mathbf{e}\right\rangle \mathrm{d} \mathbf{x} \neq 0,
$$

for any nodal basis function $\phi_{\boldsymbol{\xi}} \in \mathcal{S}_{0}^{1}(\mathcal{T})$ at $\boldsymbol{\xi}$, and assume $(1.14)$ for $\phi^{\star \star}(\cdot)$. Then, the solution $\left(u_{h}, \mathbf{m}_{h}, \lambda_{h}\right)$ of $\left(\mathbf{P}_{\varepsilon, N}^{\ell}\right), \ell \in\{\mathrm{A}, \mathrm{B}\}$ is unique for $\ell=\mathrm{B}$ but not for $\ell=\mathrm{A}$, in general. Moreover, provided that the solution of $(\mathbf{P})$ satisfies $\mathbf{m} \in H(\operatorname{div}, \omega)$ (for $\ell=\mathrm{A}$ ) or $\mathbf{m} \in H^{1}(\omega)^{d}$ (for $\left.\ell=\mathrm{B}\right)$, there holds

$$
\begin{gathered}
\left\|\nabla\left(u-u_{h}\right)\right\|_{L^{2}(\Omega)}+\left\|D \phi^{\star \star}(\mathbf{m})-D \phi^{\star \star}\left(\mathbf{m}_{h}\right)\right\|_{L^{2}(\omega)}+\left\|\lambda \mathbf{m}-\lambda_{h} \mathbf{m}_{h}\right\|_{L^{2}(\omega)}+\left\|\left(\beta_{\mathcal{E}} h_{\mathcal{E}}\right)^{1 / 2}\left[\mathbf{m}-\mathbf{m}_{h}\right]\right\|_{2 ;\left.\mathcal{E}\right|_{\omega}} \\
\leq C\left(\|\varepsilon \lambda\|_{L^{2}(\omega)}^{2}+\left\|\mathbf{f}-\mathbf{f}_{\mathcal{T}}\right\|_{L^{2}(\omega)}^{2}+\left\|\lambda \mathbf{m}-(\lambda \mathbf{m})_{\mathcal{T}}\right\|_{L^{2}(\omega)}^{2}+\left\|u-I_{\mathcal{T}}^{1} u\right\|_{L^{2}(\omega)}\right. \\
\left.+\left\|\nabla\left(u-I_{\mathcal{T}}^{1} u\right)\right\|_{L^{2}(\Omega)}^{2}+\sum_{E \in \mathcal{E}} \frac{1}{h_{E}} \int_{E}\left|u-I_{\mathcal{T}}^{1} u\right|^{2} \mathrm{~d} \mathbf{x}+\left\|\mathbf{m}-\mathbf{m}_{\mathcal{T}}\right\|_{L^{2}(\omega)}^{2}+\left\|\left(\beta_{\mathcal{E}} h_{\mathcal{E}}\right)^{1 / 2}\left[\mathbf{m}-\mathbf{m}_{\mathcal{T}}\right]\right\|_{2 ;\left.\mathcal{E}\right|_{\omega}}\right)^{1 / 2} .
\end{gathered}
$$

\section{Remark 1.2.}

(1) If we suppose $(u, \mathbf{m}, \lambda \mathbf{m}) \in H_{0}^{1}(\Omega) \cap H^{2}(\Omega) \times H^{1}(\omega) \times H^{1}(\omega)^{d}$, the right hand side is of order $\mathcal{O}(\varepsilon+h)$, which favours $\varepsilon=\mathcal{O}\left(h_{\mathcal{T}}\right)$. According to Theorem 2.1(b) below, the nonconforming method in [5] converges at same rate - whereas a conforming discretization without stabilization does not share this property [5].

(2) Inverse-type inequalities enter the proof which restrict applicability to quasiuniform meshes; in particular, this implies existence of a uniform constant $C>0$, s.t. by trace-inequality

$$
\frac{1}{C} \sum_{E \in \mathcal{E}} \frac{1}{h_{E}} \int_{E}\left|u-I_{\mathcal{T}}^{1} u\right|^{2} \mathrm{~d} \mathbf{x} \leq\left\|\nabla\left(u-I_{\mathcal{T}}^{1} u\right)\right\|_{L^{2}(\Omega)}^{2}+\left\|h_{\mathcal{T}}^{-1}\left(u-I_{\mathcal{T}}^{1} u\right)\right\|_{L^{2}(\omega)}^{2},
$$

and

$$
\frac{1}{C} \sum_{E \in \mathcal{E}} \beta_{E} h_{E} \int_{E}\left|\mathbf{m}-\mathbf{m}_{\mathcal{T}}\right|^{2} \mathrm{~d} \mathbf{x} \leq\left\|h_{\mathcal{T}} \nabla \mathbf{m}\right\|_{L^{2}(\omega)}^{2}+\left\|\mathbf{m}-\mathbf{m}_{\mathcal{T}}\right\|_{L^{2}(\omega)}^{2} .
$$

(3) The analysis for the nonconforming method in [5] is much more technical, using discrete Helmholtzdecomposition principle $(d=2)$, and is more involved for general domains $\omega \subset \mathbb{R}^{d}$.

(4) Condition (1.15) can easily be realized in practice, using e.g. a "corner element" at $\boldsymbol{\xi} \in \partial \omega$.

Computational experiments are reported in Section 4 to illustrate our theoretical results. These studies aim at shedding light at the following controversial subjects:

- comparison of the conforming, non-conforming and both stabilized conforming methods $\left(\mathbf{P}_{h_{\mathcal{T}, N}, N}\right)$, $\ell \in\{\mathrm{A}, \mathrm{B}\}$

- mutual dependence of the choices of penalization and stabilization parameter;

- performance of the stabilization strategies $\left(\mathbf{P}_{h_{\mathcal{T}}, N}^{\ell}\right), \ell \in\{\mathrm{A}, \mathrm{B}\}$ and some slight modifications in two and three spatial dimensions.

As a summary, the computational experiments evidence broad flexibility of both stabilization ansatzes $\left(\mathbf{P}_{h_{\mathcal{T}}, N}^{\ell}\right)$, $\ell \in\{\mathrm{A}, \mathrm{B}\}$ with respect to different choices of $\beta_{E}=\mathcal{O}(1)$, and optimal convergence behavior; academic examples in Section 4 where $|\nabla \mathbf{m}|$ is large compared to moderate values of $|\operatorname{div} \mathbf{m}|$ show better performance of $\left(\mathbf{P}_{h_{\mathcal{T}}, N}^{\mathrm{A}}\right)$ 
if compared to $\left(\mathbf{P}_{h_{\mathcal{T}}, N}^{\mathrm{B}}\right)$. Moreover, experiments indicate a slight reduction of performance of both stabilizations $\left(\mathbf{P}_{h_{T}, N}^{\ell}\right), \ell \in\{\mathrm{A}, \mathrm{B}\}$ on non-quasiuniform meshes.

One motivation for computational micromagnetism is the practical difficulty to physically observe internal magnetization pattern of 3D-ferromagnetic specimen [8]. A proper numerical tool to efficiently reduce complexity of 3D numerical (FE-based) studies are adaptively refined meshes according to a posteriori error analysis which is presented in Section 3; derived error indicators to control local mesh refinement are based on local residuals and jump information for computed magnetization $\mathbf{m}_{h}: \omega \rightarrow \mathbb{R}^{d}$ across interfaces $\left.E \subset \mathcal{E}\right|_{\omega}$, as well as jumps of the stray-field $\nabla u_{h}: \Omega \rightarrow \mathbb{R}^{d}$ across faces of elements $E \subset \mathcal{E}$. It will be clear from Theorem 3.1 and Remark 3.1 that a posteriori error control cannot meet both, reliability and efficiency at the same time - which is plausible from the strongly nonlinear character of Problem (P) and limited regularity of solutions; see Section 4 for further discussion in this direction.

The static model above can describe quasistatic evolution of soft magnetic materials with sufficient accuracy, but cannot be used to study hysteresis behavior of hard ferromagnets where the thickness of the loop is significant. Hysteretic behavior can be modeled by Landau-Lifshitz-Gilbert equation [12,17], which is a mesoscopic (phenomenological) model to describe spin dynamics behavior on submicron spatial scales across no more than some nanoseconds [13]; in contrast, macroscopic (phenomenological) models describe evolutionary behavior in ferromagnetic bulk specimen up to millimeter size on time-scales of much larger magnitude. Recently, a new evolutionary, rate-independent macroscopic model to describe hysteresis losses based on plasticity models in metals and shape-memory alloys has been proposed by Kružík and Roubíček [14]: here, dissipation/hysteretic effects are described in terms of evolution of the configuration, $t \mapsto \mathbf{q}(t) \equiv(\boldsymbol{\nu}(t), \mathbf{m}(t))$, where at positive times configurations

$$
\mathbf{q}(t) \in \mathcal{Q}=\left\{(\boldsymbol{\nu}(t), \mathbf{m}(t)) \in \overline{\mathcal{A}} \times L^{2}(\omega)^{d} \mid \mathbf{m}(\mathbf{x}, t)=\int_{\mathcal{S}^{d-1}} \mathbf{A} \nu_{\mathbf{x}}(t)(\mathrm{d} \mathbf{A})\right\} \subset L_{\mathrm{w}}^{\infty}\left(\omega ; \mathcal{M}\left(\mathcal{S}^{d-1}\right)\right) \times L^{2}(\omega)^{d},
$$

are governed by the following first-order evolution inclusion,

$$
\partial R\left(\mathbf{q}_{t}\right)+D \bar{E}(\mathbf{q})+N_{\mathcal{Q}}(\mathbf{q}) \ni \mathbf{f} \otimes \mathbf{I d}, \quad \mathbf{q}(0)=\mathbf{q}_{0} \in \mathcal{Q},
$$

where $\partial R$ denotes the subdifferential of $R\left(\mathbf{q}_{t}\right) \equiv R\left(\nu_{t}, \mathbf{m}_{t}\right):=\int_{\omega}\left|\xi \bullet \nu_{t}\right| \mathrm{d} \mathbf{x}$, for a given function $\xi: \mathcal{S}^{d-1} \rightarrow \mathbb{R}$ that reflects a dissipation mechanism during pole transition, and $N_{\mathcal{Q}}(\mathbf{q})$ the normal cone to $\mathcal{Q}$ at $\mathbf{q}$. The operation " $\otimes$ " is defined for any $\mathbf{h} \in L^{2}(\omega)^{d}$ and any $\mathbf{S} \in C\left(\mathcal{S}^{d-1}\right)^{d}$, by $[\mathbf{h} \otimes \mathbf{S}](\mathbf{x}, \mathbf{A})=\sum_{i=1}^{d} h_{i}(\mathbf{x}) S_{i}(\mathbf{A})$. Also, $\mathbf{q}^{0} \equiv\left(\nu_{0}, \mathbf{m}_{0}\right)$ is the initial configuration; hence, one has to set up initial volume fractions additional to the given momentum $\mathbf{m}_{0}$. By using the inherent energy balance, solutions to (1.16) are constructed in [14] as certain limits of existing solutions $\mathbf{q}^{j} \in \mathcal{Q}, j \geq 0$ of the implicit Euler discretization

$$
\partial R\left(\frac{\mathbf{q}^{j}-\mathbf{q}^{j-1}}{\Delta t}\right)+D \bar{E}\left(\mathbf{q}^{j}\right)+N_{\mathcal{Q}}\left(\mathbf{q}^{j}\right) \ni \mathbf{f}^{j} \otimes \mathbf{I d}, \quad \mathbf{q}^{0}=\mathbf{q}_{0}, \quad j \geq 1 .
$$

In a next step, a spatial discretization of (1.17) is numerically solved by using an active set strategy for selected contributing atoms $\left\{\mathbf{A}_{T, i}\right\}_{T, i} \subset \mathcal{S}^{d-1}$ to compute $\nu_{h}=\sum_{\left.T \in \mathcal{T}\right|_{\omega}} \sum_{i \geq 0} \alpha_{T, i} \delta_{\mathbf{A}_{T, i}}, c f$. [10,14]. If $\xi: \mathcal{S}^{d-1} \rightarrow \mathbb{R}$ is supposed to be affine, $(1.16)$ may be rewritten as $(c f .[10,14])$

$$
\partial R^{* *}\left(\mathbf{m}_{t}\right)+D E^{* *}(\mathbf{m})+N_{\mathcal{A}^{* *}}(\mathbf{m}) \ni \mathbf{f}, \quad \mathbf{m}(0)=\mathbf{m}^{0},
$$

for $R^{* *}\left(\mathbf{m}_{t}\right):=\int_{\omega}\left|\xi\left(\mathbf{m}_{t}\right)\right| \mathrm{d} \mathbf{x}$; if compared to (1.16), the advantage of (1.18) is that the evolutionary problem is formulated in physical quantities, i.e., in the macroscopic magnetization rather than in Young measures. For subsequent applications, we choose $\xi(\mathbf{m})=H_{c}\langle\mathbf{m}, \mathbf{e}\rangle$, where $H_{c}>0$ is the so-called "coercive force"; our choice of $\xi$ is in accordance with the experimental observation that the thickness of the hysteresis loop vanishes if we magnetize the specimen in a direction perpendicular to the easy axis $\mathbf{e} \in \mathcal{S}^{d-1}$. A discretization which 


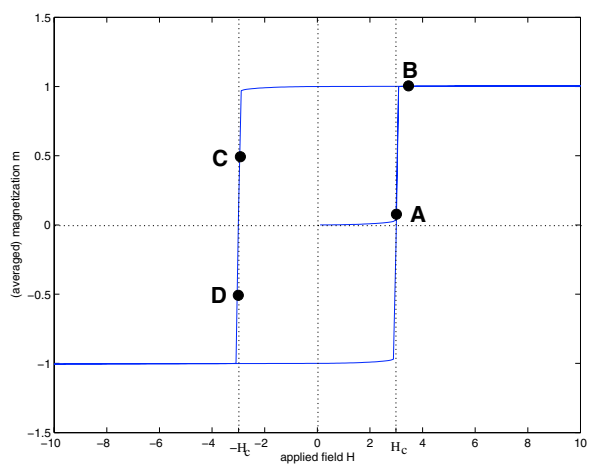

FiguRE 1. Hysteresis loop for an applied field $\left\{\mathbf{f}_{h}^{j}\right\}_{j}$ vs. averaged magnetization $\frac{1}{|\omega|} \int_{\omega}\left\langle\mathbf{m}_{h}^{j}, \mathbf{e}\right\rangle \mathrm{d} \mathbf{x}$ in Example 4.5, using $\left(\mathbf{P}_{h_{\mathcal{T}}, N}^{\mathrm{A}}\right)$; compare with Figure 15.

uses implicit Euler method leads to the following (regularized) problem: for every $j=1, \ldots, T / \tau$, minimize $E_{\delta ; j}^{* *}: \mathcal{A}^{* *} \rightarrow \mathbb{R}$, where

$$
E_{\delta ; j}^{* *}(\mathbf{m})=\int_{\omega} \phi^{* *}(\mathbf{m}) \mathrm{d} \mathbf{x}+\frac{1}{2} \int_{\Omega}|\nabla u|^{2} \mathrm{~d} \mathbf{x}-\int_{\omega}\left\langle\mathbf{f}^{j}, \mathbf{m}\right\rangle \mathrm{d} \mathbf{x}+\int_{\omega} \sqrt{\left(\xi(\mathbf{m})-\xi\left(\mathbf{m}^{j-1}\right)\right)^{2}+\delta} \mathrm{d} \mathbf{x}, \quad \delta \geq 0
$$

for $\mathbf{f}^{j}(\cdot)=\mathbf{f}(\cdot, j \tau)$, subject to (1.4). For $\phi \in C^{1}$, minimizers are characterized as solutions to the Euler-Lagrange equation

$$
\begin{aligned}
& \nabla u+D \phi^{* *}(\mathbf{m})+\frac{\xi(\mathbf{m})-\xi\left(\mathbf{m}^{j-1}\right)}{\sqrt{\left|\xi(\mathbf{m})-\xi\left(\mathbf{m}^{j-1}\right)\right|^{2}+\delta}} D \xi(\mathbf{m})+\lambda \mathbf{m}=\mathbf{f}^{j} \quad \text { a.e. in } \omega, \\
& \int_{\Omega}\langle\nabla u, \nabla w\rangle \mathrm{d} \mathbf{x}=\int_{\omega}\langle\mathbf{m}, \nabla w\rangle \mathrm{d} \mathbf{x} \quad \forall w \in H_{0}^{1}(\Omega), \\
& 0 \leq \lambda, \quad|\mathbf{m}| \leq 1, \text { and } \lambda(1-|\mathbf{m}|)_{+}=0 \quad \text { a.e. in } \omega .
\end{aligned}
$$

As we will see later, the shape of corresponding hysteresis loops strongly depends on choices of $\delta$ (see Fig. 17). The last term in (1.19) is derived from a (regularized) approximation of $\int_{(j-1) \tau}^{j \tau} \int_{\omega}\left|\xi\left(\mathbf{m}_{t}\right)\right| \mathrm{d} \mathbf{x} \mathrm{d} t$. The choice $\delta=\mathcal{O}\left(\tau^{2}\right)$ is motivated by

$$
\begin{aligned}
\int_{(j-1) \tau}^{j \tau}\left|\xi\left(\mathbf{m}_{t}\right)\right| \mathrm{d} t & \approx \int_{(j-1) \tau}^{j \tau} \sqrt{\left|\xi\left(\mathbf{m}_{t}(t)\right)\right|^{2}+c_{\delta}} \mathrm{d} t \\
& \approx \int_{(j-1) \tau}^{j \tau} \sqrt{\left|\xi\left(\frac{\mathbf{m}(t)-\mathbf{m}(t-\tau)}{\tau}\right)\right|^{2}+c_{\delta}} \mathrm{d} t \approx \sqrt{|\xi(\mathbf{m}(j \tau))-\xi(\mathbf{m}((j-1) \tau))|^{2}+c_{\delta} \tau^{2}}
\end{aligned}
$$

with $c_{\delta}>0$, where we used linearity of $\xi$.

\section{Remark 1.3.}

1. Since $H_{c}$ in $\xi(\mathbf{m})=H_{c}\langle\mathbf{m}, \mathbf{e}\rangle$ is kept constant, the used model (1.20)-(1.22) is not correct for modelling a virgin magnetization curve. Modifications, allowing virgin magnetization are discussed in [15].

2. A numerical discretization of (1.20)-(1.22), using a stable nonconforming penalized mixed method in the spirit of problem (3.5)-(3.7) in [5], is studied in [12], which uses $\delta=\mathcal{O}(h)$.

In Example 4.5, we use the concept of a discrete penalized, stabilized problem $\left(\mathbf{P}_{\varepsilon, N}^{\ell}\right), \ell \in\{\mathrm{A}, \mathrm{B}\}$ in this context to illustrate flexibility of the stabilization approach. 

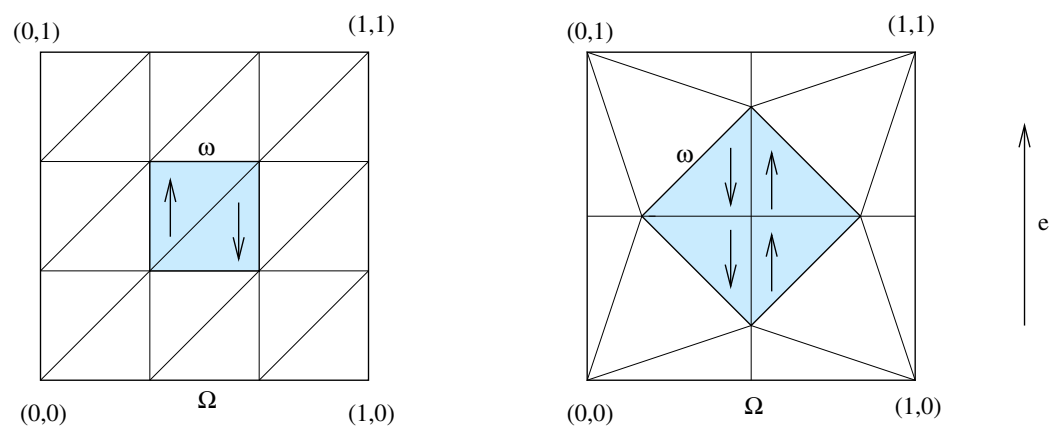

Figure 2. Non-uniqueness: spurious solutions $\mathbf{M}_{h} \in \mathcal{L}^{0}\left(\left.\mathcal{T}\right|_{\omega}\right)^{2}$ for the (a) conforming method (aligned mesh) and (b) the stabilized method $\left(\mathbf{P}_{\varepsilon, N}^{\mathrm{A}}\right)$ (rotated cube).

In this paper, we use the following notations: $L^{p}(\Omega)$ and $H^{s}(\Omega):=W^{s, 2}(\Omega)$ are standard Lebesgue and Sobolev spaces endowed with norms $\|\cdot\|_{p ; \Omega}:=\|\cdot\|_{L^{p}(\Omega)}$ and $\|\cdot\|_{s, 2, \Omega}$ for a bounded Lipschitz domain $\Omega \subset \mathbb{R}^{d}$ with boundary $\Gamma$. Let $\|\cdot\|_{k, 2 ; \omega}:=\|\cdot\|_{H^{k}(\omega)}$ and $|\cdot|_{H^{k}(\omega)}$ denote the norm and semi-norm in $H^{k}(\omega)$ for $\omega \subset \Omega$ and an integer $k$, respectively.

Let $\mathcal{T}=\{T\}$ denote a regular triangulation of $\Omega$, and $\omega$ is covered exactly by the sub-triangulation $\left.\mathcal{T}\right|_{\omega}=\{T \in \mathcal{T}: T \subset \bar{\omega}\}$. The set $\mathcal{E}$ gathers all element faces $E \subset \partial T$, with $\left.T \in \mathcal{T}\right|_{\omega}$ and $\left.\left(h_{\mathcal{E}}\right)\right|_{E}=h_{E}=\operatorname{diam}(E)$, and $\left.[\phi]\right|_{E}=\phi^{+}-\phi^{-}$across $E \in \mathcal{E}$, where $\mathbf{n}_{E} \in \mathcal{S}^{d-1}$ denotes its fixed unit normal, and $\left.\mathbf{n}_{\mathcal{E}}\right|_{E}=\mathbf{n}_{E}$. Let

$$
\begin{aligned}
\mathcal{L}^{0}(\mathcal{T}) & :=\left\{V \in L^{\infty}(\Omega): \forall T \in \mathcal{T},\left.V\right|_{T} \text { constant }\right\} \\
\mathcal{S}^{1}(\mathcal{T}) & :=\left\{V \in C(\Omega): \forall T \in \mathcal{T},\left.V\right|_{T} \text { affine }\right\}, \quad \mathcal{S}_{0}^{1}(\mathcal{T}):=\left\{V \in \mathcal{S}^{1}(\mathcal{T}): V=0 \text { on } \partial \Omega\right\}
\end{aligned}
$$

Define $\mathbf{f}_{\mathcal{T}} \in \mathcal{L}^{0}\left(\left.\mathcal{T}\right|_{\omega}\right)^{d}$ by $\left.\mathbf{f}_{\mathcal{T}}\right|_{T}=\frac{1}{T T} \int_{T} \mathbf{f} \mathrm{d} \mathbf{x}$, where $|T|$ denotes the area of $T \in \mathcal{T} . P_{\mathcal{T}}^{0}: L^{2}(\omega)^{d} \rightarrow \mathcal{L}^{0}\left(\left.\mathcal{T}\right|_{\omega}\right)^{d}$ resp. $I_{\mathcal{T}}^{0}: L^{2}(\omega)^{d} \rightarrow \mathcal{L}^{0}\left(\left.\mathcal{T}\right|_{\omega}\right)^{d}$, and $P_{\mathcal{T}}^{1}: H_{0}^{1}(\Omega) \rightarrow \mathcal{S}_{0}^{1}(\mathcal{T})$ resp. $I_{\mathcal{T}}^{1}: H_{0}^{1}(\Omega) \rightarrow \mathcal{S}_{0}^{1}(\mathcal{T})$ stand for standard projectors and (generalized) interpolation operators. The number of degrees of freedom $N=\operatorname{dim}\left(\mathcal{S}_{0}^{1}\right)+2 \operatorname{dim}\left(\mathcal{L}^{0}\left(\left.\mathcal{T}\right|_{\omega}\right)\right)$ serves as a reference to the spatial discretization $\mathcal{T}$.

\section{A priori ERror analysis for the Stabilized $\operatorname{schemes}\left(\mathbf{P}_{\varepsilon, N}^{\ell}\right), \ell \in\{\mathrm{A}, \mathrm{B}\}$.}

We start this section with observations concerning stability of the discrete models $\left(\mathbf{P}_{\varepsilon, N}^{\ell}\right), \ell \in\{\mathrm{A}, \mathrm{B}\}$. This can best be done for uniaxial ferromagnets where $\phi^{\star \star}(\mathbf{m})=\frac{1}{2}\left|\left\langle\mathbf{m}, \mathbf{e}_{\perp}\right\rangle\right|^{2}$, since uniqueness of the solution of problem $(\mathbf{P})$ is known from [5].

Let us recall results that have been obtained in [5] for the conforming method (i.e., $\beta_{\mathcal{E}} \equiv 0$, in (1.9)-(1.11)) and the nonconforming method (using Crouzeix-Raviart elements for the potential $u: \Omega \rightarrow \mathbb{R}$ ) for $d=2$. Firstly, the conforming method is shown to give solutions that are non-unique, in general, and statements upon well-posedness depend on the triangulation of $\Omega$ : Figure 2 a shows a function $\mathbf{M}_{h}: \omega \rightarrow \mathbb{R}^{2}$, where $\left|\mathbf{M}_{h}\right|=1$ a.e., such that every $\left(0, \gamma \mathbf{M}_{h}\right) \in \mathcal{S}_{0}^{1}(\mathcal{T}) \times \mathcal{L}^{0}\left(\left.\mathcal{T}\right|_{\omega}\right)^{d},-1<\gamma<1$ solves the discrete problem with $\mathbf{f} \equiv 0$. We refer to Example 1.1 in [5] for further details.

In contrast, the nonconforming method gives unique solutions for general triangulations, which favour this method over the conforming discretization. The key tool to verify uniqueness is the discrete Helmholtzdecomposition principle; it is also the key tool which leads to optimal error estimates for the nonconforming method. The following theorem summarizes these observations. 
Theorem 2.1 (from [5]). Suppose that $\phi^{\star \star}(\cdot)$ satisfies $(1.14)$ and $(\mathbf{P})$ is solved by $(u, \mathbf{m}, \lambda)$, for $d=2$. Then, (a) for the conforming penalized discretization, and a solution $\left(u_{h}, \mathbf{m}_{h}, \lambda_{h}\right) \in \mathcal{S}_{0}^{1}(\mathcal{T}) \times \mathcal{L}^{0}\left(\left.\mathcal{T}\right|_{\omega}\right)^{2} \times \mathcal{L}^{0}\left(\left.\mathcal{T}\right|_{\omega}\right)$,

$$
\begin{aligned}
& \left\|\nabla\left(u-u_{h}\right)\right\|_{L^{2}(\Omega)}+\left\|D \phi^{\star \star}(\mathbf{m})-D \phi^{\star \star}\left(\mathbf{m}_{h}\right)\right\|_{L^{2}(\omega)}+\left\|\lambda \mathbf{m}-\lambda_{h} \mathbf{m}_{h}\right\|_{L^{2}(\omega)} \\
& \leq C\left(\|\varepsilon \lambda\|_{L^{2}(\omega)}^{2}+\left\|\mathbf{f}-\mathbf{f}_{\mathcal{T}}\right\|_{L^{2}(\omega)}^{2}+\left\|\mathbf{m}-\mathbf{m}_{\mathcal{T}}\right\|_{L^{2}(\omega)}^{2}+\left\|\lambda \mathbf{m}-(\lambda \mathbf{m})_{\mathcal{T}}\right\|_{L^{2}(\omega)}^{2}\right. \\
& \left.+\left\|\nabla\left(u-I_{\mathcal{T}}^{1} u\right)\right\|_{L^{2}(\Omega)}^{2}+\left\|\mathbf{m}-\mathbf{m}_{h}\right\|_{L^{2}(\omega)}\left\|\nabla\left(u-I_{\mathcal{T}}^{1} u\right)\right\|_{L^{2}(\omega)}\right)^{1 / 2} ;
\end{aligned}
$$

(b) for the nonconforming penalized discretization, and the solution $\left(u_{h}, \mathbf{m}_{h}, \lambda_{h}\right) \in \mathcal{S}_{0}^{1, N C}(\mathcal{T}) \times \mathcal{L}^{0}\left(\left.\mathcal{T}\right|_{\omega}\right)^{2} \times \mathcal{L}^{0}\left(\left.\mathcal{T}\right|_{\omega}\right)$,

$$
\begin{aligned}
\left\|\nabla_{\mathcal{T}}\left(u-u_{h}\right)\right\|_{L^{2}(\Omega)}+\left\|D \phi^{\star \star}(\mathbf{m})-D \phi^{\star \star}\left(\mathbf{m}_{h}\right)\right\|_{L^{2}(\omega)}+\left\|\lambda \mathbf{m}-\lambda_{h} \mathbf{m}_{h}\right\|_{L^{2}(\omega)} & \\
\leq C\left(\|\varepsilon \lambda\|_{L^{2}(\omega)}^{2}+\left\|\mathbf{f}-\mathbf{f}_{\mathcal{T}}\right\|_{L^{2}(\omega)}^{2}\right. & +\left\|\mathbf{m}-\mathbf{m}_{\mathcal{T}}\right\|_{L^{2}(\omega)}^{2}+\left\|\lambda \mathbf{m}-(\lambda \mathbf{m})_{\mathcal{T}}\right\|_{L^{2}(\omega)}^{2} \\
& \left.+\left\|\nabla_{\mathcal{T}}\left(u-I_{\mathcal{T}}^{1, N C} u\right)\right\|_{L^{2}(\Omega)}^{2}+\left\|h_{\mathcal{T}} \nabla_{\mathcal{T}}\left(\mathbf{m}-\nabla_{\mathcal{T}} u\right)\right\|_{L^{2}(\Omega)}^{2}\right)^{1 / 2} .
\end{aligned}
$$

\section{Remark 2.1.}

(1) For the used notation in the nonconforming setting, see [5].

(2) If we suppose $(u, \mathbf{m}, \lambda \mathbf{m}) \in H_{0}^{1}(\Omega) \cap H^{2}(\Omega) \times H^{1}(\omega)^{2} \times H^{1}(\omega)^{2}$, the right hand side in (a) is bounded by $\mathcal{O}(\varepsilon+\sqrt{h})$, for $h=\max _{\mathcal{T}} h_{\mathcal{T}}$, whereas the upper bound in (b) is of magnitude $\mathcal{O}(\varepsilon+h)$. The bottleneck in (a) to obtain this bound is the last term in the brackets that cannot be controlled effectively, see Example 3.1 in [5] for theoretical justification of this matter.

One may attribute deterioration of order of convergence for the conforming method to non-uniqueness of the computed solution and pollution by unphysical oscillatory structures. In contrast, however, our studies for the stabilization techniques involving $t_{\ell}(\cdot, \cdot), \ell \in\{\mathrm{A}, \mathrm{B}\}$ show that these issues are not necessarily connected: $\left(\mathbf{P}_{\varepsilon, N}^{\mathrm{B}}\right)$ is well-defined, provided that (1.14) holds, but not $\left(\mathbf{P}_{\varepsilon, N}^{\mathrm{A}}\right)$. Both methods give an optimal convergence rate $\mathcal{O}(\varepsilon+h)$, provided that $(u, \mathbf{m}, \lambda \mathbf{m}) \in H_{0}^{1}(\Omega) \cap H^{2}(\Omega) \times H^{1}(\omega)^{d} \times H^{1}(\omega)^{d}$.

We start with a counterexample for $\left(\mathbf{P}_{\varepsilon, N}^{\mathrm{A}}\right)$ that excludes uniqueness of $\left(u_{h}, \mathbf{m}_{h}, \lambda_{h}\right)$, in general.

Example 2.1. Let $\Omega=(0,1)^{2} \subset \mathbb{R}^{2}, \omega=\left\{\mathbb{R}^{2} \ni \mathbf{z}:=(x, y):\left|\mathbf{z}-\left(\frac{1}{2}, \frac{1}{2}\right)\right|_{l_{1}}<\frac{1}{4}\right\}$, and $\mathcal{T}$ given as depicted in Figure 2b. It can easily be seen that $\mathcal{L}^{0}\left(\left.\mathcal{T}\right|_{\omega}\right)^{2} \ni \mathbf{M}_{h}=\gamma \operatorname{sign}\left(x-\frac{1}{2}\right) \mathbf{e}, 0 \leq \gamma<1$ solves $\left(\mathbf{P}_{\varepsilon, N}^{\mathrm{A}}\right)$ for $\phi^{\star \star}(\mathbf{m})=\frac{1}{2}\left|\left\langle\mathbf{m}, \mathbf{e}_{\perp}\right\rangle\right|^{2}$, and $\mathbf{f} \equiv 0$. Note, in particular, that $\int_{\omega}\left\langle\mathbf{M}_{h}, \nabla \phi_{h}\right\rangle \mathrm{d} \mathbf{x}=0$, for all $\phi_{h} \in \mathcal{S}_{0}^{1}(\mathcal{T})$.

This is in contrast to uniqueness for $\left(\mathbf{P}_{\varepsilon, N}^{\mathrm{B}}\right)$.

Lemma 2.1. Let (1.14) and (1.15) be valid, and $\mathbf{e} \in \mathcal{S}^{d-1}, d=2,3$. Then, Problem $\left(\mathbf{P}_{\varepsilon, N}^{\mathrm{B}}\right)$ has a unique solution.

Proof. Let $\left(u_{h}^{i}, \mathbf{m}_{h}^{i}, \lambda_{h}^{i}\right), i=1,2$ be two solutions of $\left(\mathbf{P}_{\varepsilon, N}^{\mathrm{B}}\right)$, we obtain

$$
\begin{array}{r}
\left\|\nabla\left(u_{h}^{1}-u_{h}^{2}\right)\right\|_{L^{2}(\Omega)}^{2}+\left\|D \phi^{* *}\left(\mathbf{m}_{h}^{2}\right)-D \phi^{* *}\left(\mathbf{m}_{h}^{1}\right)\right\|_{L^{2}(\omega)}^{2}+\left(\lambda_{h}^{1} \mathbf{m}_{h}^{1}-\lambda_{h}^{2} \mathbf{m}_{h}^{2}, \mathbf{m}_{h}^{1}-\mathbf{m}_{h}^{2}\right) \\
+t_{\mathrm{B}}\left(\left[\mathbf{m}_{h}^{1}-\mathbf{m}_{h}^{2}\right],\left[\mathbf{m}_{h}^{1}-\mathbf{m}_{h}^{2}\right]\right)=0 .
\end{array}
$$

The third term is non-negative, see Theorem 3.1 in [5]. Clearly, this implies $u_{h}^{1}=u_{h}^{2} \in \mathcal{S}_{0}^{1}(\mathcal{T})$, and $\left\langle\mathbf{m}_{h}^{1}-\mathbf{m}_{h}^{2}, \mathbf{e}\right\rangle=$ const $\left.\right|_{\omega}$. Filtering out these modes via (1.15) proves the assertion.

The remainder of this section verifies Theorem 1.1 for $\left(\mathbf{P}_{\varepsilon, N}^{\ell}\right), \ell \in\{\mathrm{A}, \mathrm{B}\}$, simultaneously. Let $\left(u_{h}, \mathbf{m}_{h}, \lambda_{h}\right)$ be a solution to $\left(\mathbf{P}_{\varepsilon, N}^{\ell}\right), \ell \in\{\mathrm{A}, \mathrm{B}\}$, and $(e, \boldsymbol{\delta}):=\left(u-u_{h}, \mathbf{m}-\mathbf{m}_{h}\right) \in H_{0}^{1}(\Omega) \times L^{2}(\omega)^{d}$. For $\mathbf{m} \in H(\operatorname{div}, \omega)$ 
$(\ell=\mathrm{A})$ or $\mathbf{m} \in H^{1}(\omega)^{d}(\ell=\mathrm{B})$ infer the following equations valid for all $\left(w_{h}, \boldsymbol{\mu}_{h}\right) \in \mathcal{S}_{0}^{1}(\mathcal{T}) \times \mathcal{L}^{0}\left(\left.\mathcal{T}\right|_{\omega}\right)^{d}$,

$$
\begin{aligned}
& \left(\nabla e, \nabla w_{h}\right)_{\Omega}-\left(\boldsymbol{\delta}, \nabla w_{h}\right)_{\omega}=0, \\
& \left(\nabla e, \boldsymbol{\mu}_{h}\right)_{\omega}+\left(D \phi^{* *}(\mathbf{m})-D \phi^{* *}\left(\mathbf{m}_{h}\right), \boldsymbol{\mu}_{h}\right)_{\omega}+\left(\lambda \mathbf{m}-\lambda_{h} \mathbf{m}_{h}, \boldsymbol{\mu}_{h}\right)_{\omega}+t_{\ell}\left([\boldsymbol{\delta}],\left[\boldsymbol{\mu}_{h}\right]\right)=\left(\mathbf{f}-\mathbf{f}_{\mathcal{T}}, \boldsymbol{\mu}_{h}\right)_{\omega} .
\end{aligned}
$$

Testing with the admissible functions $\left(w_{h}, \boldsymbol{\mu}_{h}\right)=\left(u_{h}-I_{\mathcal{T}}^{1} u, \mathbf{m}_{h}-\mathbf{m}_{\mathcal{T}}\right)$, this leads to

$$
\begin{aligned}
&\|\nabla e\|_{L^{2}(\Omega)}^{2}+\left\|D \phi^{* *}(\mathbf{m})-D \phi^{* *}\left(\mathbf{m}_{h}\right)\right\|_{L^{2}(\omega)}^{2}+\left(\lambda \mathbf{m}-\lambda_{h} \mathbf{m}_{h}, \boldsymbol{\delta}\right)+t_{\ell}([\boldsymbol{\delta}],[\boldsymbol{\delta}]) \\
&=(\nabla e-\boldsymbol{\delta}, \nabla(u-\left.\left.I_{\mathcal{T}}^{1} u\right)\right)_{\Omega}+\left(\mathbf{m}-\mathbf{m}_{\mathcal{T}}, \nabla e\right)_{\omega}+\left(D \phi^{* *}(\mathbf{m})-D \phi^{* *}\left(\mathbf{m}_{h}\right), \mathbf{m}-\mathbf{m}_{\mathcal{T}}\right)_{\omega} \\
&+\left(\lambda \mathbf{m}-\lambda_{h} \mathbf{m}_{h}, \mathbf{m}-\mathbf{m}_{\mathcal{T}}\right)_{\omega}+t_{\ell}\left([\boldsymbol{\delta}],\left[\mathbf{m}-\mathbf{m}_{\mathcal{T}}\right]\right)+\left(\mathbf{f}-\mathbf{f}_{\mathcal{T}}, \mathbf{m}_{h}-\mathbf{m}_{\mathcal{T}}\right)_{\omega},
\end{aligned}
$$

since $\int_{T}\left\langle\mathbf{m}_{h}-\mathbf{m}_{\mathcal{T}}, \mathbf{f}-\mathbf{f}_{\mathcal{T}}\right\rangle \mathrm{d} \mathbf{x}=0$.

We deal with the terms on the right hand side of (2.1) independently. Firstly, the convexity of the indicator function $\psi$ in $(\mathbf{P})$ and the penalization in $\left(\mathbf{P}_{\varepsilon, N}^{\ell}\right)$ yield the estimate

$$
\frac{\varepsilon}{2}\left|\mathbf{m}_{h}\right|^{2}\left(\lambda_{h}\right)^{2} \leq\left\langle\lambda \mathbf{m}-\lambda_{h} \mathbf{m}_{h}, \mathbf{m}-\mathbf{m}_{h}\right\rangle+\frac{\varepsilon}{2} \lambda^{2} .
$$

Secondly, applying integration by parts on each element $\left.T \in \mathcal{T}\right|_{\omega}$ in the first term on the right hand side of (2.1) causes

$$
\begin{aligned}
\left|\left(\boldsymbol{\delta}, \nabla\left(u-I_{\mathcal{T}}^{1} u\right)\right)_{\omega}\right| & \leq\left|\left(\operatorname{div} \mathbf{m}, u-I_{\mathcal{T}}^{1} u\right)_{\omega}\right|+\left|\sum_{E \in \mathcal{E}} \int_{E}\langle\boldsymbol{\delta}, \mathbf{n}\rangle\left(u-I_{\mathcal{T}}^{1} u\right) \mathrm{d} \mathbf{x}\right| \\
& \leq C\left\|u-I_{\mathcal{T}}^{1} u\right\|_{L^{2}(\omega)}+\frac{1}{4} t_{\ell}([\boldsymbol{\delta}],[\boldsymbol{\delta}])+\sum_{E \in \mathcal{E}} \frac{1}{h_{E}} \int_{E}\left|u-I_{\mathcal{T}}^{1} u\right|^{2} \mathrm{~d} \mathbf{x},
\end{aligned}
$$

due to continuity of discrete potential functions. We can now benefit from standard interpolation results (for traces) and a trace inequality to deal with the last term effectively.

Thirdly, in order to bound the fourth term on the right hand side of (2.1), we start testing (2.1) with $\boldsymbol{\mu}_{h}=(\lambda \mathbf{m})_{\mathcal{T}}-\lambda_{h} \mathbf{m}_{h}$ and employ an inverse-type estimate for $t_{\ell}^{1 / 2}\left(\left[\boldsymbol{\mu}_{h}\right],\left[\boldsymbol{\mu}_{h}\right]\right)$ to obtain a constant $C>0$, such that

$$
\begin{aligned}
\left\|\lambda \mathbf{m}-\lambda_{h} \mathbf{m}_{h}\right\|_{L^{2}(\omega)} \leq & C\left(\left\|\lambda \mathbf{m}-(\lambda \mathbf{m})_{\mathcal{T}}\right\|_{L^{2}(\omega)}+\|\nabla e\|_{L^{2}(\omega)}+\left\|D \phi^{* *}(\mathbf{m})-D \phi^{* *}\left(\mathbf{m}_{h}\right)\right\|_{L^{2}(\omega)}\right. \\
& \left.+t_{\ell}^{1 / 2}([\boldsymbol{\delta}],[\boldsymbol{\delta}])+\left\|\mathbf{f}-\mathbf{f}_{\mathcal{T}}\right\|_{L^{2}(\omega)}\right)
\end{aligned}
$$

Now, unknown terms in (2.3) can be absorbed on the left hand side of (2.1), where Young's inequality is used for the fourth term on the right hand side. Hence, we have

$$
\begin{aligned}
\| \nabla e & \left\|_{L^{2}(\Omega)}^{2}+\right\| D \phi^{* *}(\mathbf{m})-D \phi^{* *}\left(\mathbf{m}_{h}\right)\left\|_{L^{2}(\omega)}^{2}+\int_{\omega} \frac{\varepsilon}{2}\left|\mathbf{m}_{h}\right|^{2}\left(\lambda_{h}\right)^{2} \mathrm{~d} \mathbf{x}+\right\|\left(\beta_{\mathcal{E}} h_{\mathcal{E}}\right)^{1 / 2}\left[\mathbf{m}-\mathbf{m}_{h}\right] \|_{2 ; \mathcal{E} \mid \omega}^{2} \\
\leq & C\left(\left\|u-I_{\mathcal{T}}^{1} u\right\|_{L^{2}(\omega)}+\sum_{E \in \mathcal{E}} \frac{1}{h_{E}} \int_{E}\left|u-I_{\mathcal{T}}^{1} u\right|^{2} \mathrm{~d} \mathbf{x}+\left\|\nabla\left(u-I_{\mathcal{T}}^{1} u\right)\right\|_{L^{2}(\Omega)}^{2}\right. \\
& \left.+\left\|\mathbf{m}-I_{\mathcal{T}}^{0} \mathbf{m}\right\|_{L^{2}(\omega)}^{2}+\left\|(\lambda \mathbf{m})-(\lambda \mathbf{m})_{\mathcal{T}}\right\|_{L^{2}(\omega)}^{2}+\left\|\mathbf{f}-\mathbf{f}_{\mathcal{T}}\right\|_{L^{2}(\omega)}^{2}\right)+\int_{\omega} \frac{\varepsilon}{2} \lambda^{2} \mathrm{~d} \mathbf{x}=: B .
\end{aligned}
$$

Finally, a bootstrapping argument helps to effectively control the right hand side of (2.4) with respect to the last term, by using

$$
\left(\lambda_{h}\right)^{2}\left|\mathbf{m}_{h}\right|^{2}-\lambda^{2}|\mathbf{m}|^{2} \leq\left(\lambda_{h}\left|\mathbf{m}_{h}\right|+\lambda|\mathbf{m}|\right)\left|\lambda_{h} \mathbf{m}_{h}-\lambda \mathbf{m}\right|,
$$


and hence, by using (2.4), and choosing $\gamma>0$ sufficiently small,

$$
\left|\int_{\omega} \frac{\varepsilon}{2}\left(\left(\lambda_{h}\right)^{2}\left|\mathbf{m}_{h}\right|^{2}-\lambda^{2}|\mathbf{m}|^{2}\right) \mathrm{d} \mathbf{x}\right| \leq \frac{1}{4 \gamma^{2}} \int_{\omega} \frac{\varepsilon^{2}}{2} \lambda^{2} \mathrm{~d} \mathbf{x}+\gamma^{2}\left\|\lambda \mathbf{m}-\lambda_{h} \mathbf{m}_{h}\right\|_{L^{2}(\omega)}^{2}+\varepsilon B .
$$

(Part of) The latter term can be absorbed via (2.3) on the left hand side of (2.4). This furnishes the proof of Theorem 1.1.

\section{A posteriori error analysis for the stabilized schemes $\left(\mathbf{P}_{\varepsilon, N}^{\ell}\right), \ell \in\{\mathrm{A}, \mathrm{B}\}$}

Our goal is to construct residual-based adaptive algorithms for selective mesh refinement. It is due to the strongly nonlinear character of Problem $(\mathbf{P})$ and limited regularity properties of its solution that we cannot expect a posteriori error estimates which meet both, reliability and efficiency; $c f$. Remark 3.1. In the sequel, we denote $\omega_{h}=\left\{\mathbf{x} \in \omega \mid 0<\lambda_{h}(\mathbf{x})\right\}$. Our subsequent a posteriori error analysis controls errors in terms of local residuals, jumps of the computed stray-field $\nabla u_{h}: \Omega \rightarrow \mathbb{R}^{d}$ and magnetization $\mathbf{m}_{h}: \Omega \rightarrow \mathbb{R}^{d}$, and fulfillment of the convex side-constraint.

Theorem 3.1. Suppose that $\phi^{* *}$ satisfies (1.14), that $(u, \mathbf{m}, \lambda)$ solves Problem $(\mathbf{P})$, and $\left(u_{h}, \mathbf{m}_{h}, \lambda_{h}\right)$ solves the discrete Problem $\left(\mathbf{P}_{\varepsilon, \mathrm{N}}^{\ell}\right)$, for $\ell \in\{\mathrm{A}, \mathrm{B}\}$. Let $0<\sqrt{\frac{\varepsilon}{2}}<\gamma \ll 1$. Then there exists an $\left(\gamma, \varepsilon, h_{\mathcal{T}}, h_{\mathcal{E}}\right)$-independent constant $C>0$ such that

$$
\begin{aligned}
& \left\|\nabla\left(u-u_{h}\right)\right\|_{L^{2}(\Omega)}^{2}+\left\|D \phi^{* *}(\mathbf{m})-D \phi^{* *}\left(\mathbf{m}_{h}\right)\right\|_{L^{2}(\omega)}^{2} \\
& \leq C\left\|\frac{\varepsilon}{\gamma} \lambda_{h} \mathbf{m}_{h}\right\|_{L^{2}\left(\omega_{h}\right)}^{2}+\left\|\nabla u_{h}+D \phi^{* *}\left(\mathbf{m}_{h}\right)+\lambda_{h} \mathbf{m}_{h}-\mathbf{f}_{\mathcal{T}}\right\|_{L^{1}(\omega)}\left\|\mathbf{m}-\mathbf{m}_{\mathcal{T}}\right\|_{L^{\infty}(\omega)} \\
& +C\left\|h_{\mathcal{E}}^{1 / 2}\left\langle\mathbf{m}_{h}-\nabla u_{h}, \mathbf{n}_{\mathcal{E}}\right\rangle\right\|_{2 ; \mathcal{E}}^{2}+\left\|(1+C \gamma)\left(\beta_{\mathcal{E}} h_{\mathcal{E}}\right)^{1 / 2}\left[\mathbf{m}_{h}\right]\right\|_{2 ;\left.\mathcal{E}\right|_{\omega}}^{2} \\
& +\left\|\left[\mathbf{m}_{\mathcal{T}}\right]\right\|_{\infty ; \mathcal{E} \mid \omega}\left\|\beta_{\mathcal{E}} h_{\mathcal{E}}\left[\mathbf{m}_{h}\right]\right\|_{1 ; \mathcal{E} \mid \omega}+C\left\|\gamma\left(\lambda \mathbf{m}-(\lambda \mathbf{m})_{\mathcal{T}}\right)\right\|_{L^{2}(\omega)}^{2}+\left\|(1+C \gamma)\left(\mathbf{f}-\mathbf{f}_{\mathcal{T}}\right)\right\|_{L^{2}(\omega)}^{2} \text {. }
\end{aligned}
$$

This constant $C>0$ depends on the shape of the elements in $\mathcal{T}$ but neither on their sizes nor on the data or computed solutions.

\section{Remark 3.1.}

1. The a posteriori error analysis for $\left(\mathbf{P}_{\varepsilon, N}^{N C}\right)$ in [5] is different from here, where stabilization terms in (1.10) only allow a variational form and prevent a point-/elementwise identity like (1.5). This is the reason for arising local residual terms, next to those which account for jumps. Also, the second term in the last row of (3.1) comes from this fact, and balances regularity properties of the solution against penalization effects, which are accounted for by the leading error term of right hand side of (3.1) in terms of $\gamma>0$.

2. The constraint $|\mathbf{m}| \leq 1$ a.e. in $\omega$ implies $\left\|\mathbf{m}-\mathbf{m}_{\mathcal{T}}\right\|_{L^{\infty}(\omega)} \leq 2$, whereas for $\mathbf{m} \in W^{1, \infty}(\omega)^{d}$, a standard approximation result yields $\left\|\mathbf{m}-\mathbf{m}_{\mathcal{T}}\right\|_{L^{\infty}(\omega)} \leq C\left\|h_{\mathcal{T}} \nabla \mathbf{m}\right\|_{L^{\infty}(\omega)}$.

3. The first term in the last line can be controlled by $\leq 2\left\|\beta_{\mathcal{E}} h_{\mathcal{E}}\left[\mathbf{m}_{h}\right]\right\|_{1 ;\left.\mathcal{E}\right|_{\omega}}$, for $\mathcal{T}$ quasiuniform. In the case of $\mathbf{m} \in W^{1, \infty}(\omega)^{d}$, by Poincaré's inequality $\leq C\|\nabla \mathbf{m}\|_{L^{\infty}(\omega)}\left\|\beta_{\mathcal{E}} h_{\mathcal{E}}^{2}\left[\mathbf{m}_{h}\right]\right\|_{1 ;\left.\mathcal{E}\right|_{\omega}}$. Here, we account for total jumps in both cases $\ell \in\{\mathrm{A}, \mathrm{B}\}$.

4. The reliable upper bound $(\gamma=\mathcal{O}(\sqrt{\varepsilon}))$ for the last but one term uses

$$
\frac{1}{4}\|\lambda \mathbf{m}\|_{L^{2}(\omega)}^{2} \leq\|\mathbf{f}\|_{L^{2}(\omega)^{d}}^{2}+\left\|D \phi^{* *}(\mathbf{m})\right\|_{L^{2}(\omega)^{d}}^{2}+\|\nabla u\|_{L^{2}(\Omega)}^{2} \leq C\|\mathbf{f}\|_{L^{2}(\omega)^{d}}^{2} .
$$

In case $\lambda \mathbf{m} \in W^{1,2}(\omega)^{d}$, it may then be controlled by $\leq C\left\|\gamma h_{\mathcal{T}} \nabla(\lambda \mathbf{m})\right\|_{L^{2}(\omega)^{d^{2}}}^{2}$, hence $\gamma=\mathcal{O}(1)$. Note that reliability favours choices $\gamma^{2}=\varepsilon=\mathcal{O}\left(h_{\mathcal{T}}^{2}\right)$ for $\left(\mathbf{P}_{\varepsilon, \mathrm{N}}^{\ell}\right)$ to assure optimality of above a posteriori estimate.

5 . The leading term in the third line motivates a completely discontinuous method which uses elementwise affine ansatzes for $u_{h}: \Omega \rightarrow \mathbb{R}$, with the stabilization term above replaced with $t_{\ell}\left(\left[\mathbf{m}_{h}-\nabla u_{h}\right],\left[\boldsymbol{\mu}_{h}\right]\right)$, and an additional jump term which is necessary in (1.9) to control $\nabla u_{h}$. 
Proof. We consider terms $I+\cdots+\tilde{V}+V I$ on the right hand side of $(2.1)$, where $\tilde{V}:=t_{\ell}\left(\left[\mathbf{m}_{h}\right],\left[\mathbf{m}_{h}-\mathbf{m}_{\mathcal{T}}\right]\right)$, and the last term on the left hand side of (2.1) vanishes; using Galerkin-orthogonality property for term $I$, we find

$$
\begin{aligned}
\left(\nabla e-\delta, \nabla\left(u-I_{\mathcal{T}}^{1} u\right)\right) & =\left(\nabla u_{h}-\mathbf{m}_{h}, \nabla\left(u-I_{\mathcal{T}}^{1} u\right)\right)=\int_{\mathcal{E}}\left\langle\nabla u_{h}-\mathbf{m}_{h}, \mathbf{n}_{\mathcal{E}}\right\rangle\left(u-I_{\mathcal{T}}^{1} u\right) \mathrm{d} s \\
& \leq C\|\nabla e\|_{L^{2}(\Omega)}\left\|h_{\mathcal{E}}^{1 / 2}\left\langle\nabla u_{h}-\mathbf{m}_{h}, \mathbf{n}_{\mathcal{E}}\right\rangle\right\|_{2, \mathcal{E}} .
\end{aligned}
$$

Thanks to (1.5), terms $I I, I I I, I V$, and $V I$ can be controlled by computable local residuals,

$$
I I+I I I+I V+V I \leq\left\|\nabla u_{h}+D \phi^{* *}\left(\mathbf{m}_{h}\right)+\lambda_{h} \mathbf{m}_{h}-\mathbf{f}_{h}\right\|_{L^{2}(\omega)}\left\|\mathbf{m}_{h}-\mathbf{m}_{\mathcal{T}}\right\|_{L^{2}(\omega)} .
$$

Next,

$$
\tilde{V}=t_{\ell}\left(\left[\mathbf{m}_{h}\right],\left[\mathbf{m}_{h}\right]\right)-t\left(\left[\mathbf{m}_{h}\right],\left[\mathbf{m}_{\mathcal{T}}\right]\right) \leq\left\|\left(\beta_{\mathcal{E}} h_{\mathcal{E}}\right)^{1 / 2}\left[\mathbf{m}_{h}\right]\right\|_{2 ;\left.\mathcal{E}\right|_{\omega}}^{2}+\left\|\left[\mathbf{m}_{\mathcal{T}}\right]\right\|_{\infty ;\left.\mathcal{E}\right|_{\omega}}\left\|\beta_{\mathcal{E}} h_{\mathcal{E}}\left[\mathbf{m}_{h}\right]\right\|_{1 ; \mathcal{E} \mid} .
$$

Finally, in order to control the third term on the left hand side of (2.1), we use again (2.2); instead of (2.6), and $0<\sqrt{\frac{\varepsilon}{2}}<\gamma$, we obtain

$$
\left|\int_{\omega}\left(\frac{\varepsilon}{4} \lambda_{h}^{2}\left|\mathbf{m}_{h}\right|^{2}-\lambda^{2}|\mathbf{m}|^{2}\right) \mathrm{d} \mathbf{x}\right| \leq \int_{\omega} \frac{\varepsilon^{2}}{8 \gamma^{2}} \lambda_{h}^{2}\left|\mathbf{m}_{h}\right|^{2} \mathrm{~d} \mathbf{x}+\gamma^{2}\left\|\lambda \mathbf{m}-\lambda_{h} \mathbf{m}_{h}\right\|_{L^{2}(\omega)}^{2}
$$

Upon choosing $\gamma \ll 1$, we may now employ (2.3), and the assertion is proved.

\section{Numerical EXAMPLES}

We now discuss several numerical experiments for uniaxial ferromagnets, including a comparison of methods $\left(\mathbf{P}_{h_{\mathcal{T}}, N}^{\ell}\right), \ell \in\{\mathrm{A}, \mathrm{B}\}$ to the nonconforming and the unstabilized conforming method (see [5]). For academic studies, we consider a slightly generalized version $(\tilde{\mathbf{P}})$ of Problem $(\mathbf{P})$, for general right hand side $s \in L^{2}(\Omega)$ of (1.2). The modified discrete penalized, stabilized problem $\left(\tilde{\mathbf{P}}_{h_{\mathcal{T}}, N}^{\ell} \mid \mathcal{S}_{0}^{1}(\mathcal{T}) \times \mathcal{L}^{0}\left(\left.\mathcal{T}\right|_{\omega}\right)^{d} \times \mathcal{L}^{0}\left(\left.\mathcal{T}\right|_{\omega}\right)\right)$, shortly $\left(\tilde{\mathbf{P}}_{h_{\mathcal{T}}, N}^{\ell}\right), \ell \in\{\mathrm{A}, \mathrm{B}\}$, for $\beta_{\mathcal{E}}>0$, and given $(s, \mathbf{f}) \in L^{2}(\Omega) \times L^{2}(\omega)^{d}$, reads as follows:

Seek $\left(u_{h}, \mathbf{m}_{h}, \lambda_{h}\right) \in \mathcal{S}_{0}^{1}(\mathcal{T}) \times \mathcal{L}^{0}\left(\left.\mathcal{T}\right|_{\omega}\right)^{d} \times \mathcal{L}^{0}\left(\left.\mathcal{T}\right|_{\omega}\right)$ such that for all $\left(w_{h}, \boldsymbol{\mu}_{h}\right) \in \mathcal{S}_{0}^{1}(\mathcal{T}) \times \mathcal{L}^{0}\left(\left.\mathcal{T}\right|_{\omega}\right)^{d}$,

$$
\begin{aligned}
& \int_{\Omega}\left\langle\nabla u_{h}, \nabla w_{h}\right\rangle \mathrm{d} \mathbf{x}-\int_{\omega}\left\langle\mathbf{m}_{h}, \nabla w_{h}\right\rangle \mathrm{d} \mathbf{x}=\int_{\Omega} s w_{h} \mathrm{~d} \mathbf{x}, \\
& \left(\nabla u_{h}, \boldsymbol{\mu}_{h}\right)_{\omega}+\left(\left\langle\mathbf{m}_{h}, \mathbf{e}_{\perp}\right\rangle,\left\langle\boldsymbol{\mu}_{h}, \mathbf{e}_{\perp}\right\rangle\right)_{\omega}+\left(\lambda_{h} \mathbf{m}_{h}, \boldsymbol{\mu}_{h}\right)_{\omega}+t_{\ell}\left(\left[\mathbf{m}_{h}\right],\left[\boldsymbol{\mu}_{h}\right]\right)=\left(\mathbf{f}, \boldsymbol{\mu}_{h}\right)_{\omega}, \\
& \lambda_{h}=\frac{1}{c_{\varepsilon} h_{\mathcal{T}}} \frac{\left(\left|\mathbf{m}_{h}\right|-1\right)_{+}}{\left|\mathbf{m}_{h}\right|} \quad \text { a.e. in } \omega,
\end{aligned}
$$

for choices $0<c_{\varepsilon}=\mathcal{O}(1)$. Correspondingly, we refer to $\left(\tilde{\mathbf{P}}_{h_{\mathcal{T}}, N}^{C}\right)$ and $\left(\tilde{\mathbf{P}}_{h_{\mathcal{T}}, N}^{N C}\right)$ as (unstabilized) conforming and nonconforming discretizations, respectively.

The discrete, nonlinear problems $\left(\tilde{\mathbf{P}}_{h_{\mathcal{T}}, N}^{\ell}\right),\left(\tilde{\mathbf{P}}_{h_{\mathcal{T}}, N}^{C}\right)$, and $\left(\tilde{\mathbf{P}}_{h_{\mathcal{T}}, N}^{N C}\right)$ are solved numerically by NewtonRaphson's method. If we abstract from the specific form of $\left(\tilde{\mathbf{P}}_{h_{\mathcal{T}}, N}^{\ell}\right)$ by setting

$$
\mathcal{F}\left(\left[u_{h}, \mathbf{m}_{h}\right] ;\left[w_{h}, \boldsymbol{\mu}_{h}\right]\right)=0 \quad \forall\left[w_{h}, \boldsymbol{\mu}_{h}\right] \in \mathcal{S}_{0}^{1}(\mathcal{T}) \times \mathcal{L}^{0}\left(\left.\mathcal{T}\right|_{\omega}\right)^{d}
$$

this amounts to running the following iteration, for $n \geq 1$,

$$
D \mathcal{F}\left(\left[u_{h}^{n}, \mathbf{m}_{h}^{n}\right],\left[w_{h}, \boldsymbol{\mu}_{h}\right] ;\left[u_{h}^{n}-u_{h}^{n+1}, \mathbf{m}_{h}^{n}-\mathbf{m}_{h}^{n+1}\right]\right)=\mathcal{F}\left(\left[u_{h}^{n}, \mathbf{m}_{h}^{n}\right] ;\left[w_{h}, \boldsymbol{\mu}_{h}\right]\right),
$$


for all tuples $\left(w_{h}, \boldsymbol{\mu}_{h}\right) \in \mathcal{S}_{0}^{1}(\mathcal{T}) \times \mathcal{L}^{0}\left(\left.\mathcal{T}\right|_{\omega}\right)^{d}$, until a certain tolerance is reached. This calculation is followed at each step by an algebraic update to compute $\left(u_{h}^{n+1}, \mathbf{m}_{h}^{n+1}\right)$. The implementation of the Newton-Raphson algorithm for 2D as well as 3D problems is performed in Matlab in the spirit of [1].

In the present situation and for given values $\left(u_{h}, \mathbf{m}_{h}\right), D \mathcal{F}\left(\left(\left[u_{h}, \mathbf{m}_{h}\right],\left[w_{h}, \boldsymbol{\mu}_{h}\right] ;\left[V_{h}, \mathbf{M}_{h}\right]\right)\right.$ has the form

$$
\begin{aligned}
\left(\nabla V_{h}, \boldsymbol{\mu}_{h}\right)_{\omega}+\left(\left\langle\mathbf{M}_{h}, \mathbf{e}_{\perp}\right\rangle,\left\langle\boldsymbol{\mu}_{h}, \mathbf{e}_{\perp}\right\rangle\right)_{\omega}+\left(\frac{1}{c_{\varepsilon} h_{\mathcal{T}}} \frac{\mathbb{H}\left(\left|\mathbf{m}_{h}\right|-1\right)\left\langle\mathbf{m}_{h}, \mathbf{M}_{h}\right\rangle}{\left|\mathbf{m}_{h}\right|^{3}} \mathbf{m}_{h}, \boldsymbol{\mu}_{h}\right)_{\omega} & +\left(\frac{1}{c_{\varepsilon} h_{\mathcal{T}}} \frac{\left(\mathbf{m}_{h}-1\right)_{+}}{\left|\mathbf{m}_{h}\right|} \mathbf{M}_{h}, \boldsymbol{\mu}_{h}\right)_{\omega}+t_{\ell}\left(\left[\mathbf{m}_{h}\right],\left[\boldsymbol{\mu}_{h}\right]\right)
\end{aligned}
$$

for tuples $\left(V_{h}, \mathbf{M}_{h}\right)$ satisfying $\left(\nabla V_{h}, \nabla w_{h}\right)_{\Omega}-\left(\mathbf{M}_{h}, \nabla w_{h}\right)_{\omega}=0$, for all $w_{h} \in \mathcal{S}_{0}^{1}(\mathcal{T})$. Here, $\mathbb{H}: \mathbb{R} \rightarrow \mathbb{R}+$ denotes the Heaviside function.

Remark 4.1. According to Example 2.1, solutions to $\left(\tilde{\mathbf{P}}_{h_{\mathcal{T}}, N}^{C}\right)$ are not unique, in general. In our computations, we added the term $\gamma\left(\mathbf{m}_{h}, \boldsymbol{\mu}_{h}\right)_{\omega}$ to the left hand side of (4.2) to single out one solution, setting $\gamma=10^{-6}$.

In order to approximate the right hand side in $\left(\tilde{\mathbf{P}}_{h_{\mathcal{T}}, N}^{\ell}\right), \ell \in\{\mathrm{A}, \mathrm{B}\}$ for given functions $s \in L^{2}(\Omega)$ and $\mathbf{f} \in\left(L^{2}(\omega)\right)^{d}$, we compute $\int_{\Omega} s \eta \mathrm{d} \mathbf{x}$ and $\int_{\omega} f \theta \mathrm{d} \mathbf{x}$ via a 7 -point Gaussian quadrature rule of order 6 where $\eta, \theta$ denote corresponding basis functions. The indefinite linear systems of equations is solved directly.

In the Examples 4.1, 4.3 below, the potential $u$ and the magnetization $\mathbf{m}$ are given. Hence the $L^{2}(\Omega)$-norms of $u-u_{h}, \nabla\left(u-u_{h}\right),\left\langle\mathbf{m}-\mathbf{m}_{h}, \mathbf{e}\right\rangle$, and $\left\langle\mathbf{m}-\mathbf{m}_{h}, \mathbf{e}_{\perp}\right\rangle$ can be calculated via a 7 -point quadrature rule on any triangle to run convergence studies.

Example 4.1. Consider $(\tilde{\mathbf{P}})$, for $\Omega=(-1,2)^{2}, \omega=(0,1)^{2}$, and

$$
u(x, y)=\left(x^{2}-x-2\right)\left(y^{2}-y-2\right) \quad \text { and } \quad \mathbf{m}_{k}(x, y)=\left(w_{k}(x, y), w_{k}(y, x)\right)^{T},
$$

where

$$
w_{k}(x, y):= \begin{cases}\widetilde{w}_{k}(x, y) & , \text { if } \widetilde{w}_{k}(x, y)^{2}+\widetilde{w}_{k}(y, x)^{2} \leq 1 \\ \widetilde{w}_{k}(x, y) / \sqrt{\widetilde{w}_{k}(x, y)^{2}+\widetilde{w}_{k}(y, x)^{2}} & , \text { else }\end{cases}
$$

for $\widetilde{w}_{k}(x, y):=\sin (\pi x) \sin (k \pi y)$. We choose $\lambda=0$ on $\left\{x \in \omega|| \mathbf{m}_{k} \mid<1\right\}$, and $\lambda=1$ else.

The prescribed potential $u$ is smooth and $\left|\operatorname{div} \mathbf{m}_{k}\right|$ is bounded, but $\left|\nabla \mathbf{m}_{k}\right|$ is unbounded as $k \rightarrow \infty$. Given $\mathbf{e} \in \mathcal{S}^{1}$, the right hand sides $s$ and $\mathbf{f}$ are computed by (4.1) and (4.2) from $u$ and $\mathbf{m}_{k}$ above. Given these data, we compute discrete solutions of $\left(\tilde{\mathbf{P}}_{h_{\mathcal{T}}, N}^{\ell}\right), \ell \in\{\mathrm{A}, \mathrm{B}\}$.

As initial mesh we use a partitioning of $\Omega$ into right-angled triangles of size $h=\sqrt{2} / 16$. If not stated otherwise, we always put $\beta_{\mathcal{E}}=\frac{1}{10}$ below.

Figure 3 shows the computed magnetization $\mathbf{m}_{h}$ for a meshing $\mathcal{T}$ aligned to e and obtained by $\left(\tilde{\mathbf{P}}_{h_{\mathcal{T}}, N}^{C}\right)($ left $)$ and $\left(\tilde{\mathbf{P}}_{h_{\mathcal{T}}, N}^{\mathrm{A}}\right)$ (right), where the first one shows spurious oscillations that are avoided by the stabilized analogue $(k=4)$. The approximated magnetization is depicted by vectors given in the center of each triangle and the colorbars show the modulus of magnetization.

Table 1 gathers convergence rates experimentally obtained for $u-u_{h}$ in the $H^{1}(\Omega)$-seminorm and the $L^{2}(\Omega)$-norm, for $k=4$ and both types of stabilization $\left(\tilde{\mathbf{P}}_{h_{\mathcal{T}}, N}^{\ell}\right), \ell \in\{\mathrm{A}, \mathrm{B}\}$. The computed convergence rates $\xi_{h}$ coincide with the theoretical predictions in Theorem 1.1 in both cases. Figures 4 and 5 provide a direct comparison of all numerical schemes discussed above for $\mathbf{e}=(1,0)$ and $k=4$. These results support a convergence behavior of first order for both methods $\left(\tilde{\mathbf{P}}_{h_{\mathcal{T}}, N}^{\ell}\right), \ell \in\{\mathrm{A}, \mathrm{B}\}$ for error $=\left\|\nabla\left(u-u_{h}\right)\right\|_{L^{2}(\Omega)}+\left\|\left\langle\mathbf{m}-\mathbf{m}_{h}, \mathbf{e}_{\perp}\right\rangle\right\|_{L^{2}(\omega)}$. Corresponding results hold for the conforming (unstabilized) method $\left(\tilde{\mathbf{P}}_{h_{\mathcal{T}}, N}^{C}\right)$ and the nonconforming method $\left(\tilde{\mathbf{P}}_{h_{\mathcal{T}}, N}^{N C}\right)$ as well, although shifting of corresponding curves reflect an increased number of unknowns in the latter case (slope $-1 / 2$, resp. 1 in Figures 4 and 5 corresponds to an experimental convergence rate 1, resp. 2, 

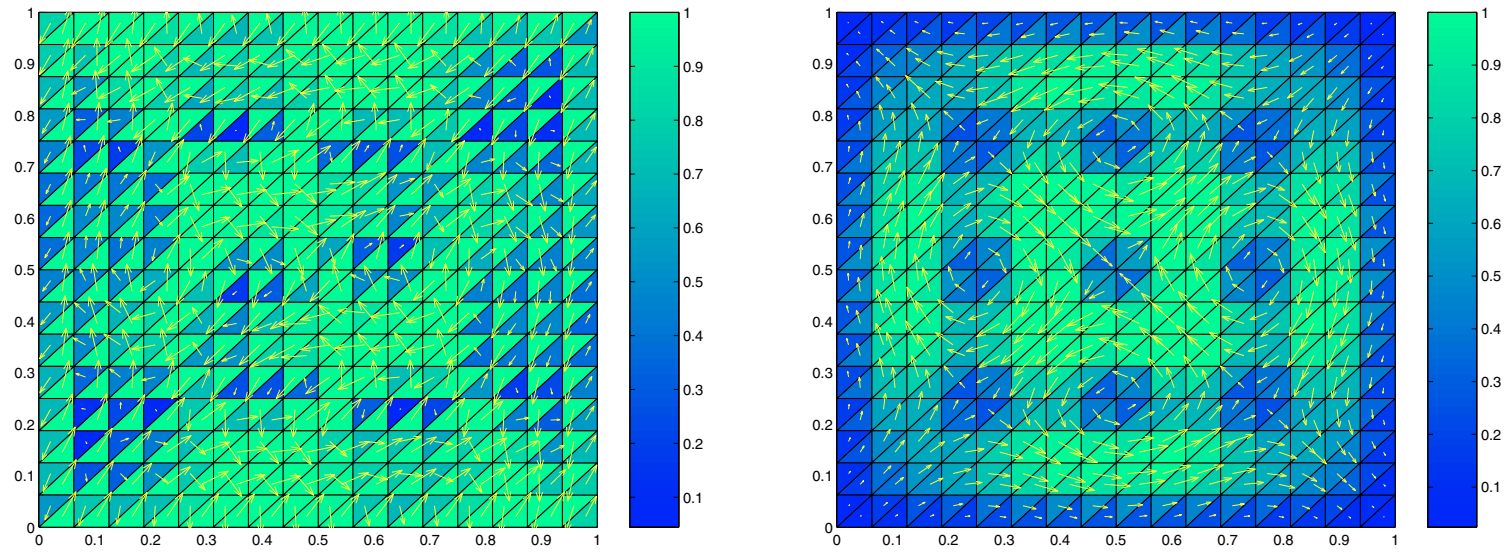

Figure 3. Plot of magnetization $\mathbf{m}_{h}$ using no stabilization (left) and stabilization $\left(\tilde{\mathbf{P}}_{h_{\mathcal{T}}, N}^{\mathrm{A}}\right)$ (right) for $\mathbf{e}=(1,0)$ and $k=4$.
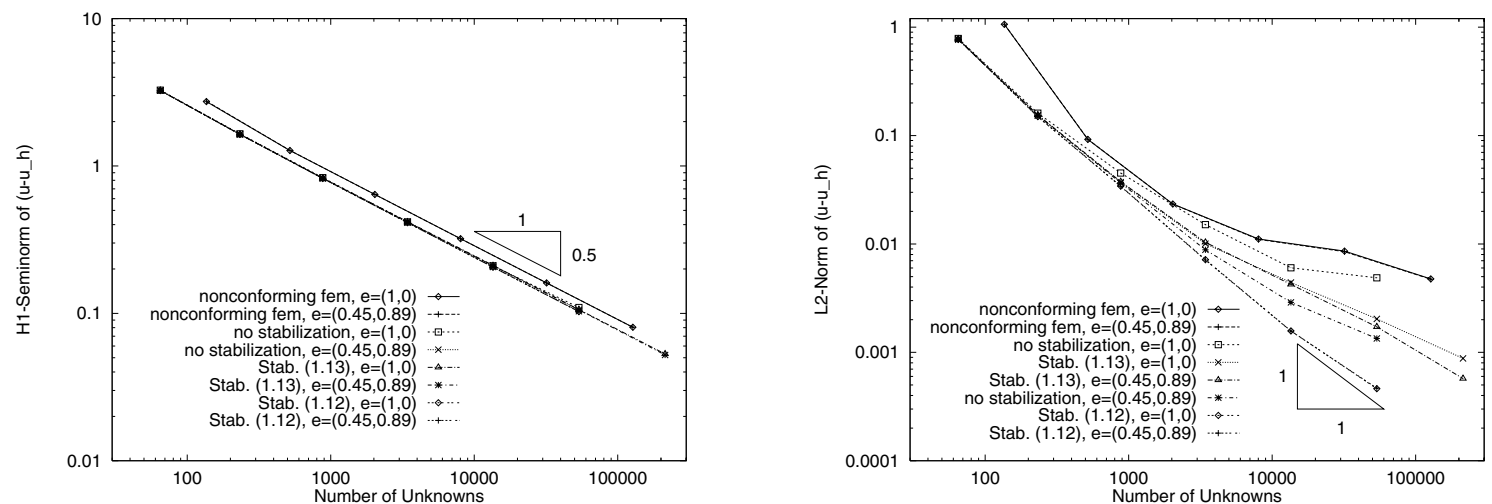

FiguRE 4. Errors $\left\|\nabla\left(u-u_{h}\right)\right\|_{L^{2}(\Omega)}$ and $\left\|u-u_{h}\right\|_{L^{2}(\Omega)}$ vs. number of unknowns $N$ in Example $4.1(k=4)$.

TABLE 1. Error $\left\|\nabla\left(u-u_{h}\right)\right\|_{L^{2}(\Omega)}$, resp. $\left\|u-u_{h}\right\|_{L^{2}(\Omega)}$ and convergence rate $\xi_{h}$, resp. $\zeta_{h}$ in Example 4.1 for $\left(\tilde{\mathbf{P}}_{h_{\mathcal{T}}, N}^{\mathrm{A}}\right)$ (left) and $\left(\tilde{\mathbf{P}}_{h_{\mathcal{T}}, N}^{\mathrm{B}}\right)$ (right) $(k=4)$.

\begin{tabular}{|c|c|c|c|c|}
\hline $\mathrm{N}$ & $\nabla\left(u-u_{h}\right)$ & $\xi_{h}$ & $\left\|u-u_{h}\right\|$ & $\zeta_{h}$ \\
\hline 65 & 3.2506 & & 0.7733 & \\
\hline 233 & 1.6377 & 103 & 0.1487 & 22 \\
\hline 881 & 0.8236 & 102 & 0.0340 & 231 \\
\hline 3425 & 0.4122 & 1.02 & 0.0071 & $\begin{array}{l}2.01 \\
2.90\end{array}$ \\
\hline 13505 & 0.2058 & 1.01 & 0.0016 & 2.20 \\
\hline 53633 & 0.1028 & 1.01 & 0.0005 & 1.10 \\
\hline
\end{tabular}

\begin{tabular}{|c|c|c|c|c|}
\hline $\mathrm{N}$ & $\nabla\left(u-u_{h}\right)$ & $\xi_{h}$ & $u-u_{h}$ & $\zeta_{h}$ \\
\hline 65 & 3.2516 & & 0.7777 & \\
\hline 233 & 1.6392 & $10^{\circ}$ & 0.1551 & \\
\hline 881 & 0.8272 & 1.00 & 0.0376 & \\
\hline 3425 & 0.4194 & $\begin{array}{l}1.00 \\
0.00\end{array}$ & 0.0104 & \\
\hline 13505 & 0.2118 & 0.99 & 0.0042 & \\
\hline 53633 & 0.1056 & 1.02 & 0.0017 & 1.0 \\
\hline
\end{tabular}

owing to $N \propto h^{-2}$ in two dimensions). As to the error $\left\|u-u_{h}\right\|_{L^{2}(\Omega)}$, improved convergence behavior of both methods $\left(\tilde{\mathbf{P}}_{h_{\mathcal{T}}, N}^{\ell}\right), \ell \in\{\mathrm{A}, \mathrm{B}\}$ over the remaining two is observed.

The present example of a mesh that is aligned to $\mathbf{e} \in \mathcal{S}^{1}$ has been chosen to reflect non-stable behavior of $\left(\tilde{\mathbf{P}}_{h_{\mathcal{T}}, N}^{C}\right)$ as already observed in [5]. This can be seen from Figure 5 (left), which shows poor convergence of 

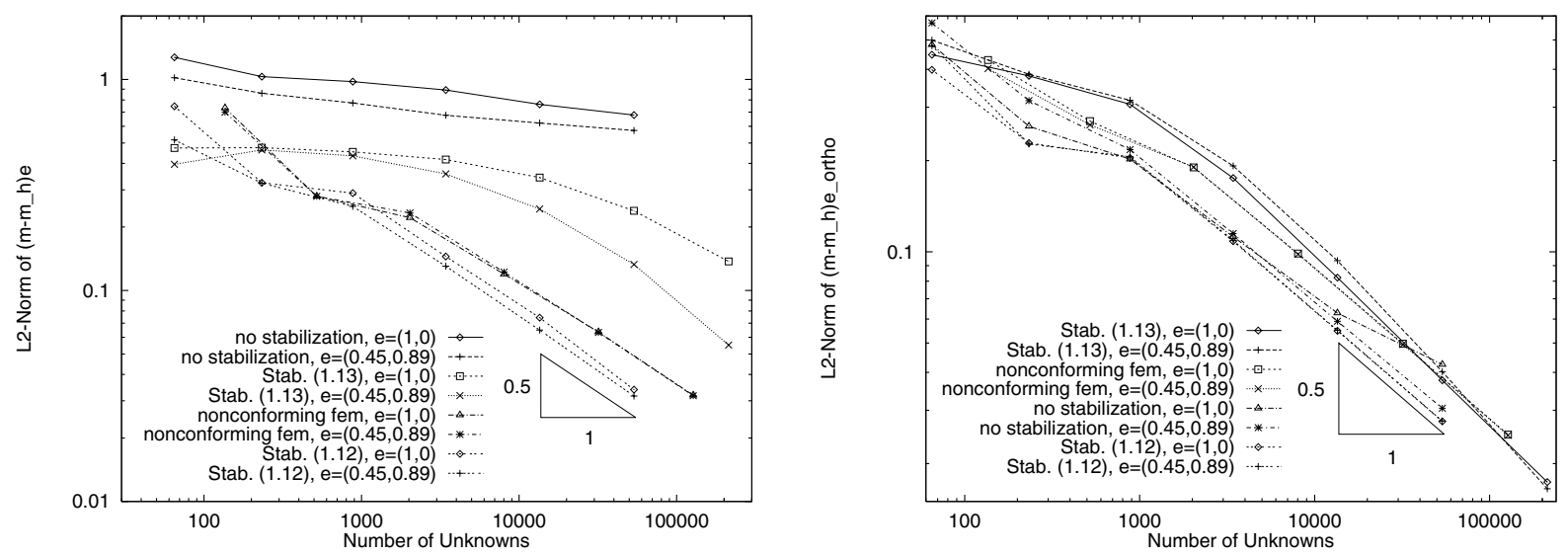

Figure 5. Errors $\left\|\left\langle\mathbf{m}-\mathbf{m}_{h}, \mathbf{e}\right\rangle\right\|_{L^{2}(\omega)}$ and $\left\|\left\langle\mathbf{m}-\mathbf{m}_{h}, \mathbf{e}_{\perp}\right\rangle\right\|_{L^{2}(\omega)}$ vs. number of unknowns $N$ in Example $4.1(k=4)$.
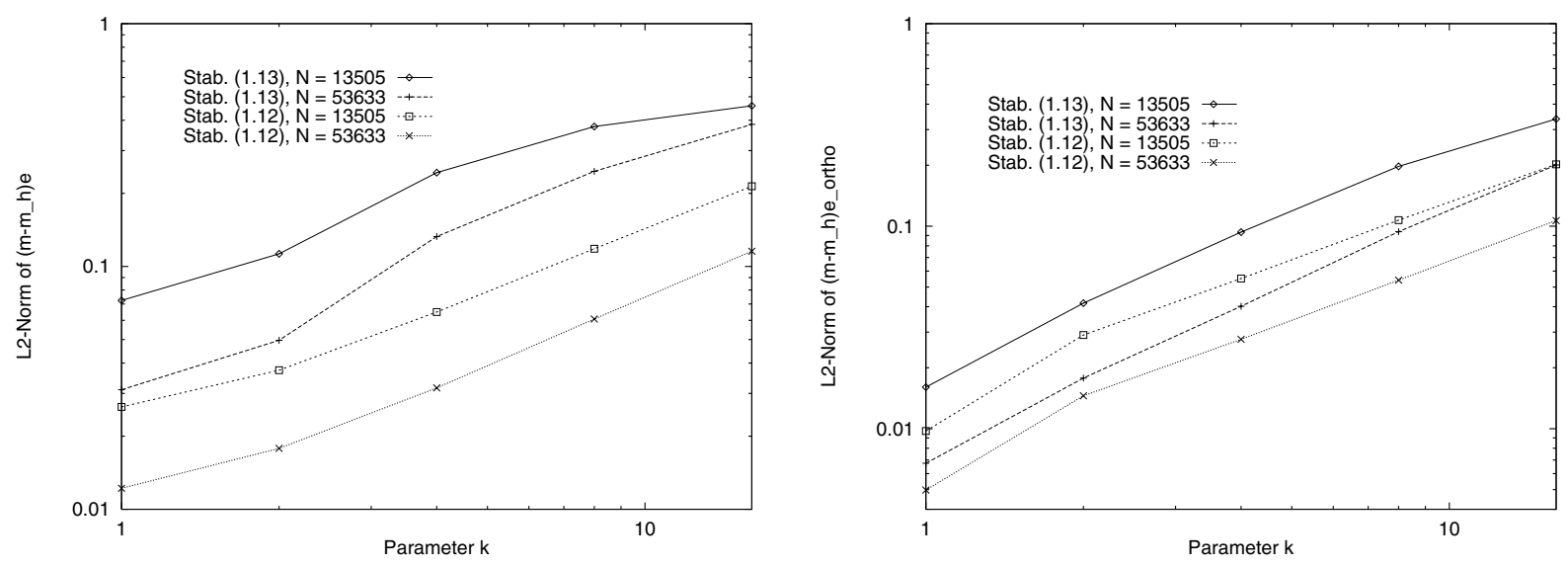

Figure 6. Errors $\left\|\left\langle\mathbf{m}-\mathbf{m}_{h}, \mathbf{e}\right\rangle\right\|_{L^{2}(\omega)}$ and $\left\|\left\langle\mathbf{m}-\mathbf{m}_{h}, \mathbf{e}_{\perp}\right\rangle\right\|_{L^{2}(\omega)}$ vs. parameter $k$ in Example 4.1.

this method. In contrast, the stabilizing effect in $\left(\tilde{\mathbf{P}}_{h_{\mathcal{T}}, N}^{\ell}\right), \ell \in\{\mathrm{A}, \mathrm{B}\}$ is evident from corresponding curves in this figure. Numerical experiments for $\left(\tilde{\mathbf{P}}_{h_{\mathcal{T}}, N}^{\mathrm{B}}\right)$ asymptotically show the same convergence behavior as is found for $\left(\tilde{\mathbf{P}}_{h_{\mathcal{T}}, N}^{\mathrm{A}}\right)$ and $\left(\tilde{\mathbf{P}}_{h_{\mathcal{T}}, N}^{N C}\right)$, but values of the error $\left\|\left\langle\mathbf{m}-\mathbf{m}_{h}, \mathbf{e}\right\rangle\right\|_{L^{2}(\omega)}$ are larger up to a factor 7 for this kind of stabilization. This observation regarding performance of $\left(\tilde{\mathbf{P}}_{h_{\mathcal{T}}, N}^{\ell}\right), \ell \in\{\mathrm{A}, \mathrm{B}\}$ has been made for different aligned and nonaligned meshes in $2 D$.

We now study the stabilized methods $\left(\tilde{\mathbf{P}}_{h_{\mathcal{T}}, N}^{\ell}\right), \ell \in\{\mathrm{A}, \mathrm{B}\}$ for highly oscillatory magnetizations. For a fixed number of unknowns $(N=13505$ and $N=53653)$, the dependence of $\left\|\left\langle\mathbf{m}-\mathbf{m}_{h}, \mathbf{e}\right\rangle\right\|_{L^{2}(\omega)}$ and $\left\|\left\langle\mathbf{m}-\mathbf{m}_{h}, \mathbf{e}_{\perp}\right\rangle\right\|_{L^{2}(\omega)}$ on the parameter $k \geq 1$ is shown in Figure 6.

Our results motivate reduced convergence behavior for $\left\|\left\langle\mathbf{m}-\mathbf{m}_{h}, \mathbf{e}_{\perp}\right\rangle\right\|_{L^{2}(\omega)}$ for $\left(\tilde{\mathbf{P}}_{h_{\mathcal{T}}, N}^{\mathrm{B}}\right)$, opposed to $\left(\tilde{\mathbf{P}}_{h_{\mathcal{T}}, N}^{\mathrm{A}}\right)$, for growing values of $k$, which illustrates higher robustness of $\left(\mathbf{P}_{h_{\mathcal{T}}, N}^{\mathrm{A}}\right)$ in the studied case.

The following two Figures 7 and 8 study the dependence of $\left\|\nabla\left(u-u_{h}\right)\right\|_{L^{2}(\Omega)},\left\|u-u_{h}\right\|_{L^{2}(\Omega)}$, $\left\|\left\langle\mathbf{m}-\mathbf{m}_{h}, \mathbf{e}\right\rangle\right\|_{L^{2}(\omega)}$, and $\left\|\left\langle\mathbf{m}-\mathbf{m}_{h}, \mathbf{e}_{\perp}\right\rangle\right\|_{L^{2}(\omega)}$ on $\beta:=\beta_{\mathcal{E}}$, for $\mathbf{e}=(2,1) / \sqrt{5}$, and $k=4$. As to $\left(\tilde{\mathbf{P}}_{h_{\mathcal{T}}, N}^{\mathrm{A}}\right)$, all considered quantities behave almost insensitive on a broad range of $\beta$, and we found optimal choices $\beta \in(0.1,1)$. A slightly more sensitive behavior can be stated for $\left(\tilde{\mathbf{P}}_{h_{\mathcal{T}}, N}^{\mathrm{B}}\right)$. 

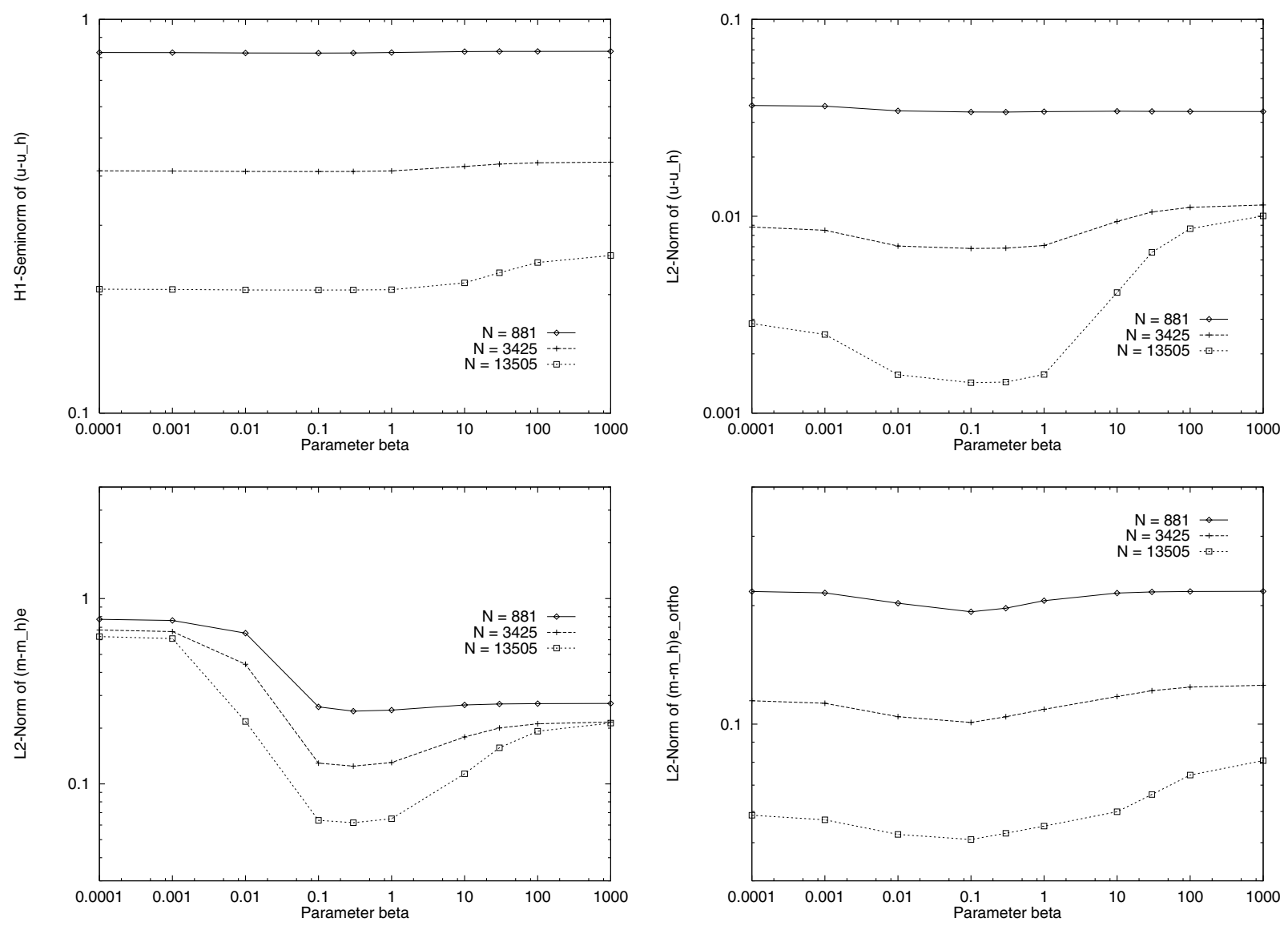

FiguRE 7. Errors vs. stabilization factor $\beta \equiv \beta_{\mathcal{E}}$ in Example 4.1 for $\left(\tilde{\mathbf{P}}_{h_{\mathcal{T}}, N}^{\mathrm{A}}\right)$.

Next, we study the effect of mesh refinement based on error indicators evidenced from Theorem 3.1; in the sequel, we set $\gamma^{2}=\varepsilon=h_{\mathcal{T}}^{2}$. Then

$$
\frac{1}{2}\left\|\nabla\left(u-u_{h}\right)\right\|_{L^{2}(\Omega)}^{2}+\left\|D \phi^{* *}(\mathbf{m})-D \phi^{* *}\left(\mathbf{m}_{h}\right)\right\|_{L^{2}(\omega)}^{2} \leq C_{\delta} \eta_{C}^{(\delta)}, \quad \delta=0,1
$$

where $C_{0}$ does not depend on $h_{\mathcal{T}}, h_{\mathcal{E}}$ but on the shape of $\mathcal{T}$,

$$
\begin{aligned}
\eta_{C}^{(0):=} & \left\|h_{\mathcal{T}} \lambda_{h} \mathbf{m}_{h}\right\|_{L^{2}\left(\omega_{h}\right)}^{2}+2\left\|\nabla u_{h}+D \phi^{* *}\left(\mathbf{m}_{h}\right)+\lambda_{h} \mathbf{m}_{h}-\mathbf{f}_{\mathcal{T}}\right\|_{L^{1}(\omega)}+\left\|\left(\beta_{\mathcal{E}} h_{\mathcal{E}}\right)^{1 / 2}\left[\mathbf{m}_{h}\right]\right\|_{2 ;\left.\mathcal{E}\right|_{\omega}}^{2} \\
& +\left\|h_{\mathcal{E}}^{1 / 2}\left\langle\mathbf{m}_{h}-\nabla u_{h}, \mathbf{n}_{\mathcal{E}}\right\rangle\right\|_{2 ;\left.\mathcal{E}\right|_{\omega}}^{2}+2\left\|\beta_{\mathcal{E}} h_{\mathcal{E}}\left[\mathbf{m}_{h}\right]\right\|_{1 ; \mathcal{E}}+\left\|\mathbf{f}-\mathbf{f}_{\mathcal{T}}\right\|_{L^{2}(\omega)}^{2}+\left\|h_{\mathcal{T}} \mathbf{f}\right\|_{L^{2}(\omega)}^{2},
\end{aligned}
$$

and $C_{1}$ additionally depends on $\|\mathbf{m}\|_{W^{1, \infty}(\omega)^{d}}$,

$$
\begin{aligned}
\eta_{C}^{(1)}:= & \left\|h_{\mathcal{T}} \lambda_{h} \mathbf{m}_{h}\right\|_{L^{2}\left(\omega_{h}\right)}^{2}+2\left\|\nabla u_{h}+D \phi^{* *}\left(\mathbf{m}_{h}\right)+\lambda_{h} \mathbf{m}_{h}-\mathbf{f}_{\mathcal{T}}\right\|_{L^{1}(\omega)}+\left\|\left(\beta_{\mathcal{E}} h_{\mathcal{E}}^{2}\right)^{1 / 2}\left[\mathbf{m}_{h}\right]\right\|_{2 ;\left.\mathcal{E}\right|_{\omega}}^{2} \\
& +\left\|h_{\mathcal{E}}^{1 / 2}\left\langle\mathbf{m}_{h}-\nabla u_{h}, \mathbf{n}_{\mathcal{E}}\right\rangle\right\|_{2 ;\left.\mathcal{E}\right|_{\omega}}^{2}+\left\|\beta_{\mathcal{E}} h_{\mathcal{E}}^{2}\left[\mathbf{m}_{h}\right]\right\|_{1 ; \mathcal{E}}+\left\|\mathbf{f}-\mathbf{f}_{\mathcal{T}}\right\|_{L^{2}(\omega)}^{2} .
\end{aligned}
$$



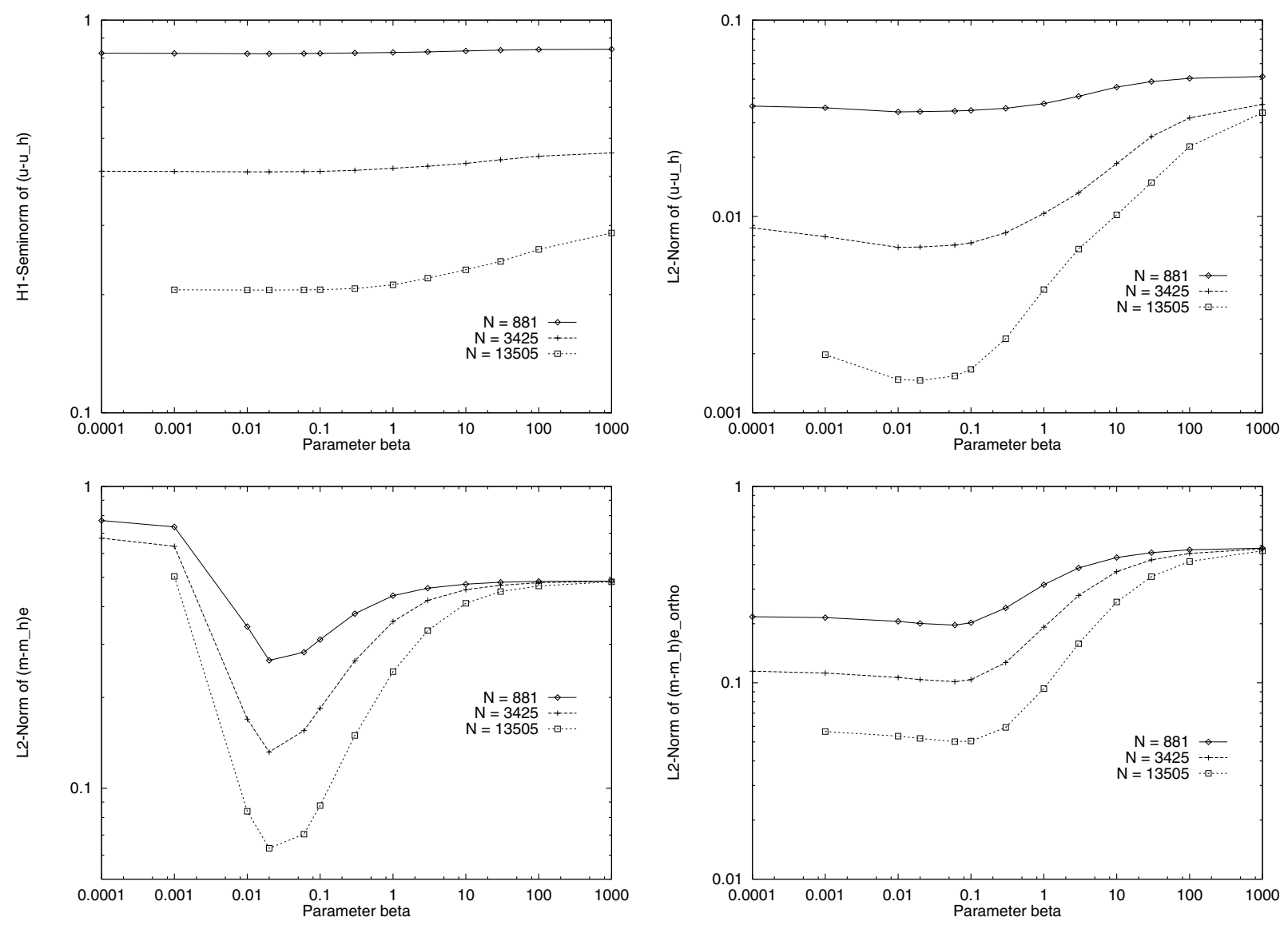

FiguRE 8. Errors vs. stabilization factor $\beta \equiv \beta_{\mathcal{E}}$ in Example 4.1 for $\left(\tilde{\mathbf{P}}_{h_{\mathcal{T}}, N}^{\mathrm{B}}\right)$.

The reason for two error estimators is based on the efficiency-reliability gap of the a posteriori analysis which is discussed in Remark 3.1. Now, (4.4), (4.5) motivate error indicators for local mesh refinement,

$$
\begin{aligned}
\eta_{C, T}^{(0)}:= & \left\|h_{\mathcal{T}} \lambda_{h} \mathbf{m}_{h}\right\|_{L^{2}(T)}^{2}+2\left\|\nabla u_{h}+D \phi^{* *}\left(\mathbf{m}_{h}\right)+\lambda_{h} \mathbf{m}_{h}-\mathbf{f}_{\mathcal{T}}\right\|_{L^{1}(T)}+\left\|\left(\beta_{\mathcal{E}} h_{\mathcal{E}}\right)^{1 / 2}\left[\mathbf{m}_{h}\right]\right\|_{2 ;\left.\mathcal{E}\right|_{\omega}}^{2} \\
& +\left\|h_{\mathcal{E}}^{1 / 2}\left\langle\mathbf{m}_{h}-\nabla u_{h}, \mathbf{n}_{\mathcal{E}}\right\rangle\right\|_{2 ; \partial T}^{2}+2\left\|\beta_{\mathcal{E}} h_{\mathcal{E}}\left[\mathbf{m}_{h}\right]\right\|_{1 ; \partial T}+\left\|\mathbf{f}-\mathbf{f}_{\mathcal{T}}\right\|_{L^{2}(T)}^{2}+\left\|h_{\mathcal{T}} \mathbf{f}\right\|_{L^{2}(T)}^{2}, \\
\eta_{C, T}^{(1)}:= & \left\|h_{\mathcal{T}} \lambda_{h} \mathbf{m}_{h}\right\|_{L^{2}(T)}^{2}+2\left\|\nabla u_{h}+D \phi^{* *}\left(\mathbf{m}_{h}\right)+\lambda_{h} \mathbf{m}_{h}-\mathbf{f}_{\mathcal{T}}\right\|_{L^{1}(T)}+\left\|\left(\beta_{\mathcal{E}} h_{\mathcal{E}}^{2}\right)^{1 / 2}\left[\mathbf{m}_{h}\right]\right\|_{2 ;\left.\mathcal{E}\right|_{\omega}}^{2} \\
& +\left\|h_{\mathcal{E}}^{1 / 2}\left\langle\mathbf{m}_{h}-\nabla u_{h}, \mathbf{n}_{\mathcal{E}}\right\rangle\right\|_{2 ; \partial T}^{2}+\left\|\beta_{\mathcal{E}} h_{\mathcal{E}}^{2}\left[\mathbf{m}_{h}\right]\right\|_{1 ; \partial T}+\left\|\mathbf{f}-\mathbf{f}_{\mathcal{T}}\right\|_{L^{2}(T)}^{2},
\end{aligned}
$$

such that $\eta_{C}^{(\delta)}=\sum_{T} \eta_{C, T}^{(\delta)}, \delta=0,1$. The subsequent mesh-refining algorithm generates a sequence $\mathcal{T}_{0}, \mathcal{T}_{1}, \ldots$ of adapted meshes.

\section{Algorithm 4.1.}

1. Start with a coarse mesh $\mathcal{T}_{0}$.

2. Solve the discrete problem with respect to $\mathcal{T}_{k}$.

3. Compute $\eta_{C, T}^{(\delta)}$ for all $T \in \mathcal{T}_{k}$.

4. Compute error bound $\eta_{C}^{(\delta)}$ and terminate or goto 5 . 

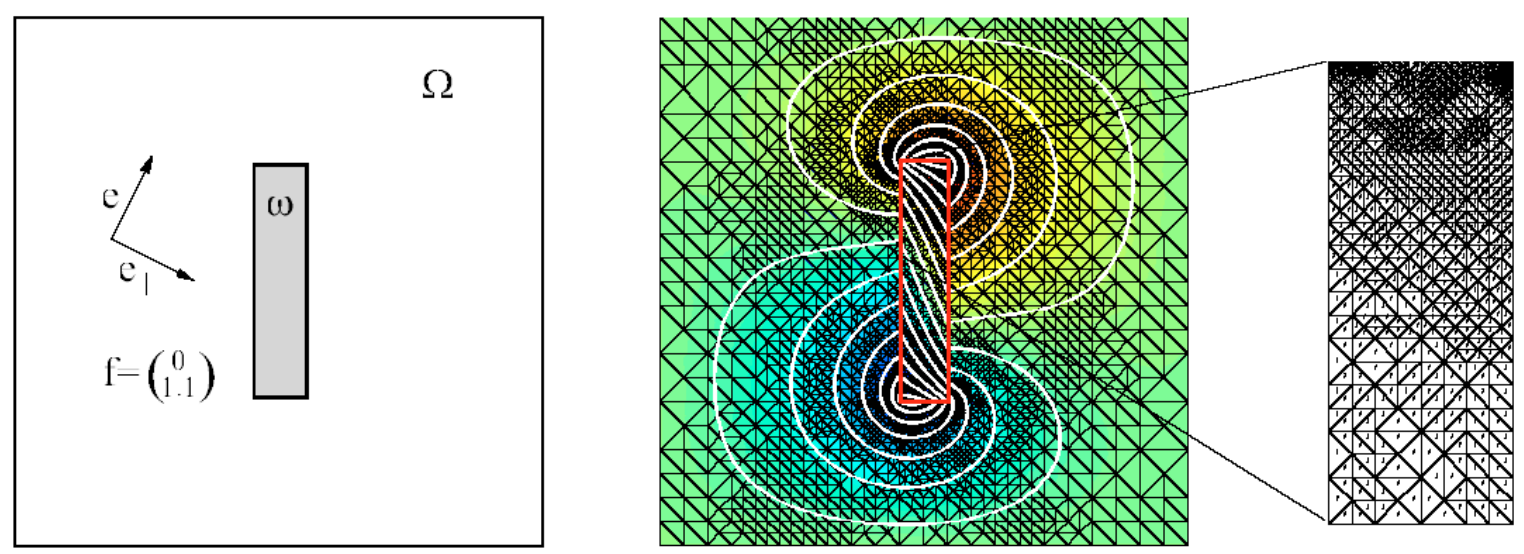

Figure 9. Configuration of Example 4.2 (left) and corresponding solution using $\left(\mathbf{P}_{h_{\mathcal{T}}, N}^{\mathrm{A}}\right)$, and adaptive mesh refinement via Alg. $4.1(\delta=1)$ (right).
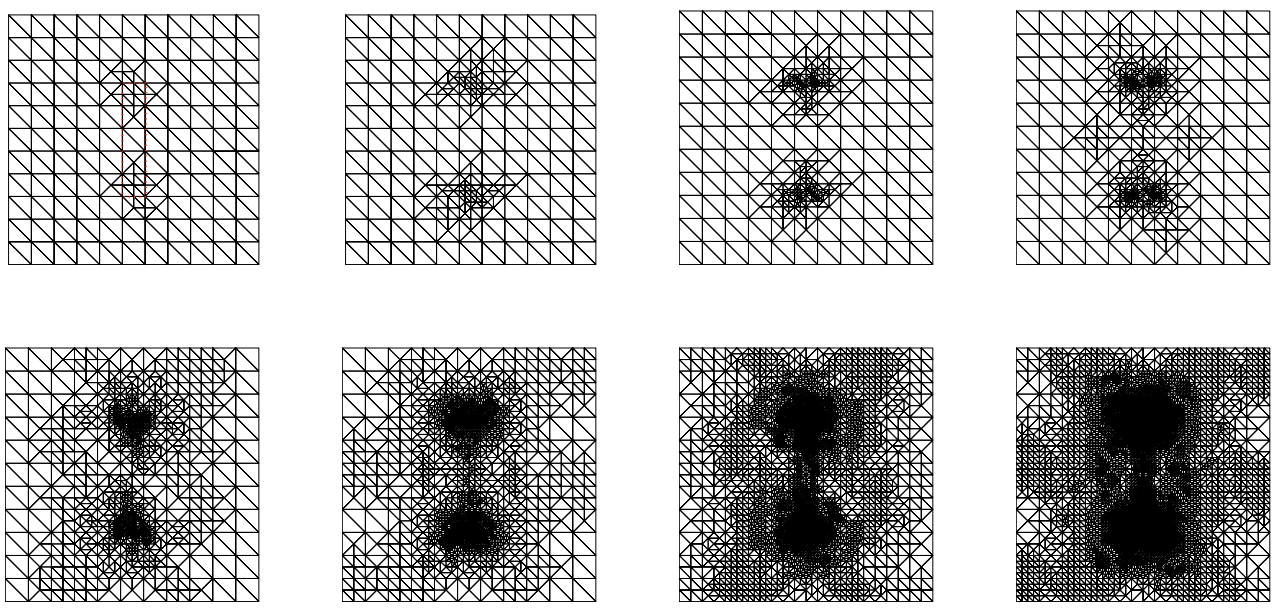

Figure 10. Sequence of adaptive meshes $\mathcal{T}_{1}, \ldots, \mathcal{T}_{8}$ using Alg. $4.1(\delta=1)$ and $\left(\mathbf{P}_{h_{\mathcal{T}}, N}^{\mathrm{A}}\right)$ in Example 4.2 .

5. Mark element $T$ red iff $\eta_{C, T}^{(\delta)} \geq \frac{1}{2} \max _{T \in \mathcal{T}_{k}} \eta_{C, T}^{(\delta)}$

6. Red-green-blue refinement (see [18]) to avoid hanging nodes, generate mesh $\mathcal{T}_{k+1}$, set $k=k+1$ and goto 2 .

The next example illustrates the effect of adaptive mesh-refinement.

Example 4.2. Let $\Omega=(-5.5,5.5)^{2}, \omega=(-0.5,0.5) \times(-2.5,2.5), \mathbf{f}=(0,1.1)^{T}$ and $\mathbf{e}=(2,1)^{T} / \sqrt{5}$ in $\left(\mathbf{P}_{h_{\mathcal{T}}, N}^{\mathrm{A}}\right)$. This problem studies potential and the magnetization in a symmetric configuration where the easy-axis $\mathbf{e}$ is not aligned to the coordinate-axis. A plot of (isolines of) the computed solution of the potential $u_{h}: \Omega \rightarrow \mathbb{R}$ on an adaptively refined mesh $\mathcal{T}_{7}$ according to Algorithm $4.1(\delta \equiv 1)$ is shown in Figure 9 (left); the corresponding computed magnetization $\mathbf{m}_{h}: \omega \rightarrow \mathbb{R}^{2}$ in the top half of $\omega$ is given in Figure 9 (right).

We use Algorithm 4.1 to generate a sequence of meshes (see Fig. 10), which refine towards the corners of $\omega$ and which show a higher mesh density in $\omega$ than outside. To assess the discretization error in the approximation 

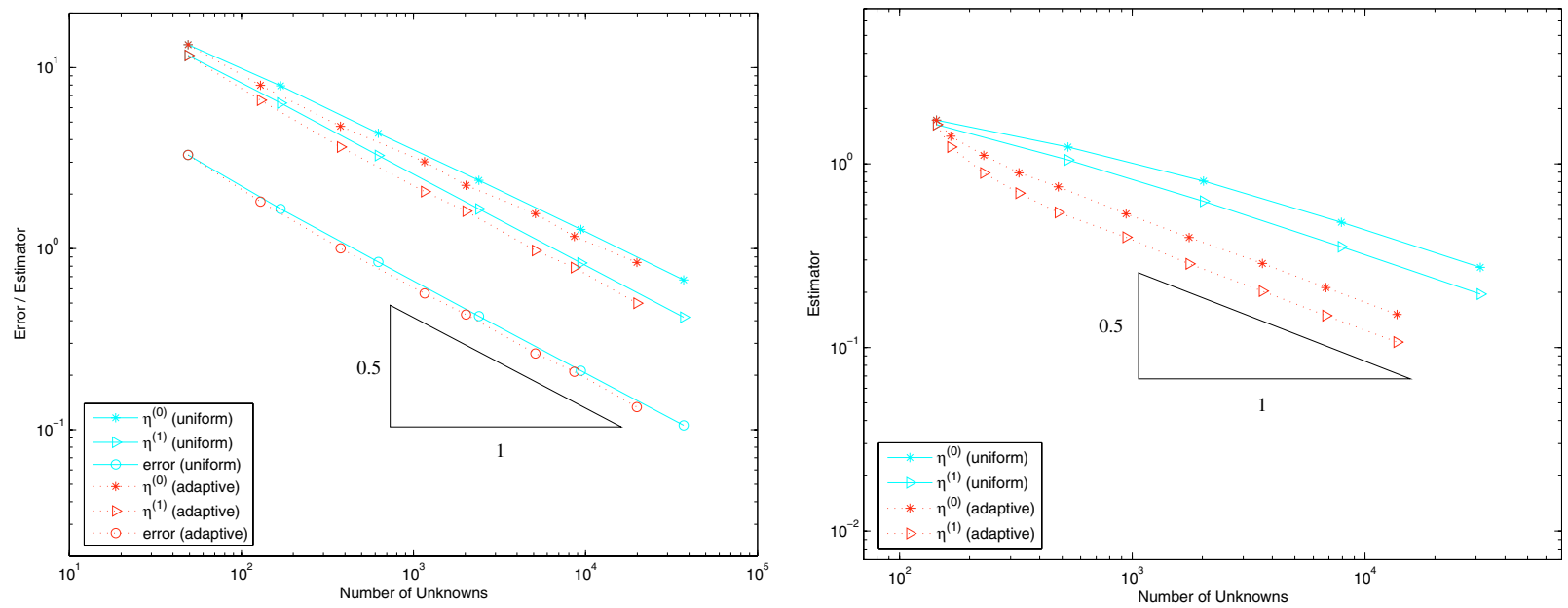

FiguRE 11. Error $\left\|\nabla\left(u-u_{h}\right)\right\|_{L^{2}(\Omega)}+\left\|D \phi^{* *}(\mathbf{m})-D \phi^{* *}\left(\mathbf{m}_{h}\right)\right\|_{L^{2}(\omega)}+\left\|\lambda \mathbf{m}-\lambda_{h} \mathbf{m}_{h}\right\|_{L^{2}(\omega)}$ (left) and error estimator $\eta_{C}^{(\delta)}, \delta=0,1$ vs. degrees of freedom $N$ for uniform and $\eta_{C}^{(1)}$-generated meshes in Example 4.1 (left) resp. Example 4.2 (right).
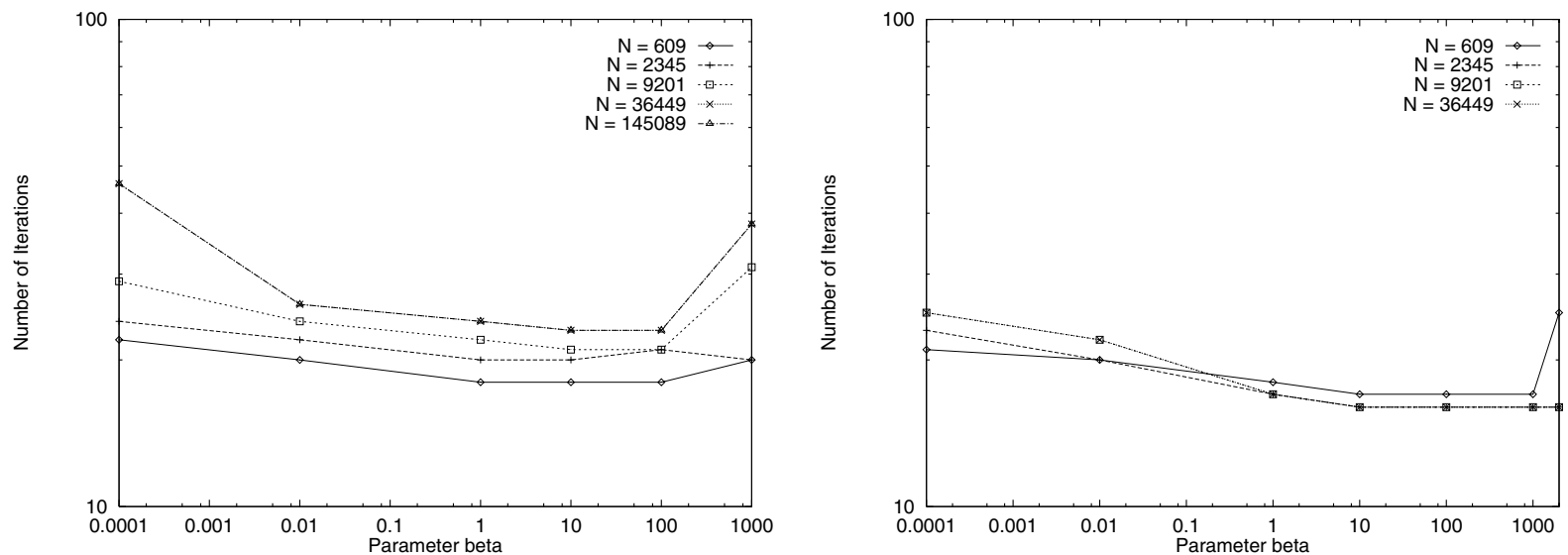

Figure 12. Number of Newton-iterations vs. number of unknowns $N$ in Example 4.1 for $\left(\tilde{\mathbf{P}}_{h_{\mathcal{T}}, N}^{\mathrm{A}}\right)$ (left) and $\left(\tilde{\mathbf{P}}_{h_{\mathcal{T}}, N}^{\mathrm{B}}\right)$ (right).

given in Figure 9, error estimators are displayed in Figure 9 for computed $\eta_{C}^{(\delta)}, \delta=0,1$. The result is plotted in Figure 11. The error estimates show the same convergence rate as the computed approximation error.

Example 4.3. We consider a 3D-example for $\left(\tilde{\mathbf{P}}_{h_{\mathcal{T}}, N}^{\ell}\right), \ell \in\{\mathrm{A}, \mathrm{B}\}$ and calculate approximations in the case of an aligned mesh $(\mathbf{e}=(0,0,1))$ and an unaligned mesh $(\mathbf{e}=(1,2,3) / \sqrt{14})$. Let $\Omega=(-1,2)^{3}$ and $\omega=(0,1)^{3}$. The right hand sides $s$ and $\mathbf{f}$ are determined from the given functions

$$
\begin{gathered}
u(x, y, z)=\left(x^{2}-x-2\right)\left(y^{2}-y-2\right)\left(z^{2}-z-2\right), \\
\mathbf{m}_{k}(x, y, z)=\frac{1}{2}\left(\begin{array}{c}
\sin (k \pi x) \sin (\pi y) \sin (\pi z) \\
\sin (\pi x) \sin (k \pi y) \sin (\pi z) \\
\sin (\pi x) \sin (\pi y) \sin (\pi z)
\end{array}\right), \quad \lambda(x, y, z)= \begin{cases}0, & \text { if }\left|\mathbf{m}_{k}(x, y, z)\right|<1 \\
1, & \text { else. }\end{cases}
\end{gathered}
$$



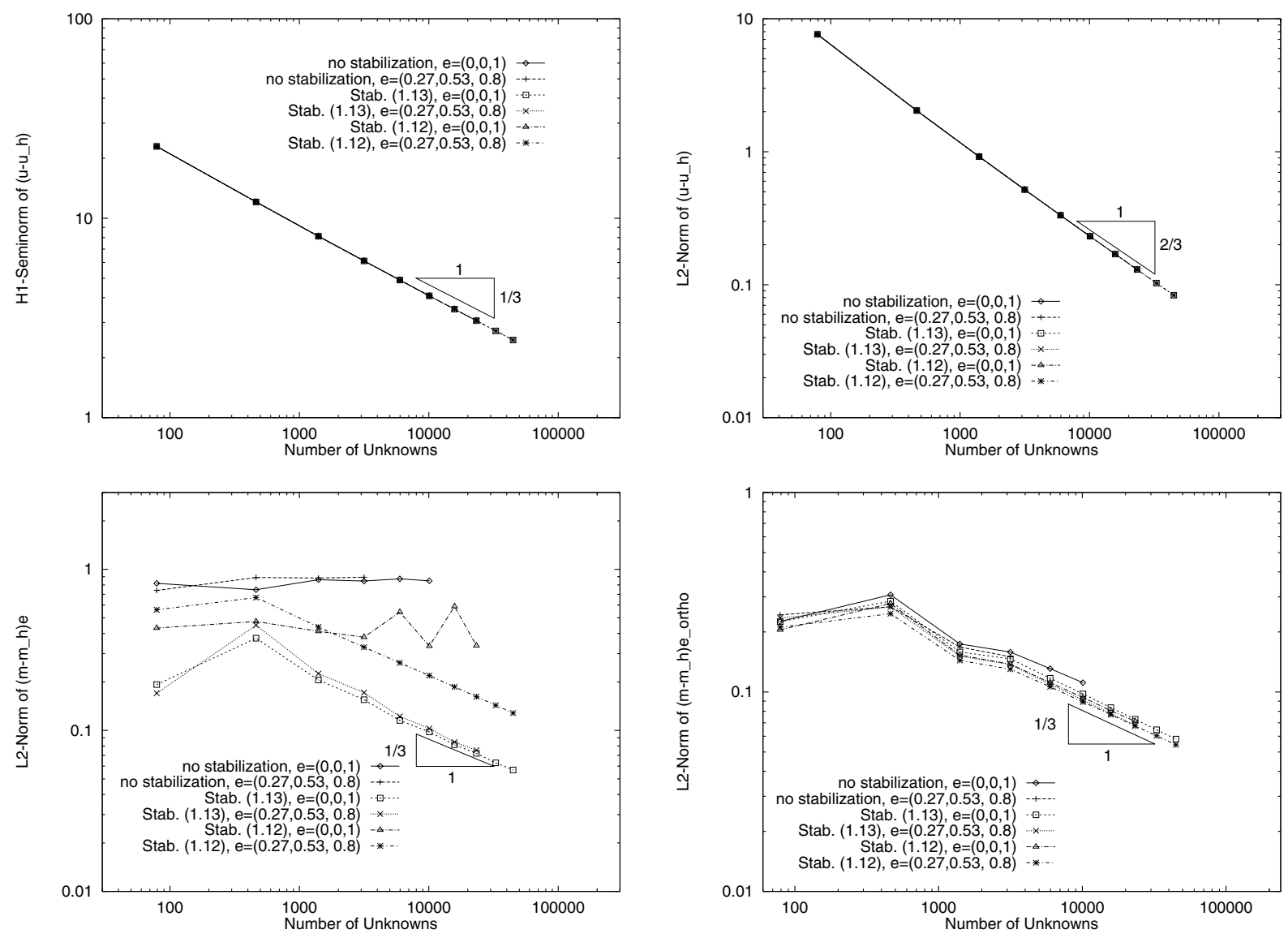

FiguRE 13. Errors $\left\|\nabla\left(u-u_{h}\right)\right\|_{L^{2}(\Omega)},\left\|u-u_{h}\right\|_{L^{2}(\Omega)}, \quad\left\|\left\langle\mathbf{m}-\mathbf{m}_{h}, \mathbf{e}\right\rangle\right\|_{L^{2}(\omega)}, \quad$ and $\left\|\left\langle\mathbf{m}-\mathbf{m}_{h}, \mathbf{e}_{\perp}\right\rangle\right\|_{L^{2}(\omega)}$ vs. number of unknowns $N$ in Example $4.3(k=3)$.

Similar to Example 4.1, the prescribed potential $u$ is smooth and $\left|\operatorname{div} \mathbf{m}_{k}\right|$ is bounded, but $\left|\nabla \mathbf{m}_{k}\right|$ is unbounded as $k$ tends to infinity.

Division of $\Omega$ into equally sized cubes and subdivision of these cubes into five tetrahedra give the uniform triangulation $\mathcal{T}$ used in our computations. To integrate quantities like $\int_{\Omega} s \eta \mathrm{d} \mathbf{x}, \int_{\omega} f \theta \mathrm{d} \mathbf{x}$, etc., we use a Gaussian quadrature rule of order $6[9]$ where $\eta, \theta$ denote basis functions.

We set $\mathbf{e}_{\perp}:=\operatorname{span}\left\{\mathbf{e}_{\perp}^{1}, \mathbf{e}_{\perp}^{2}\right\}$ and $\left\|\left\langle\mathbf{m}-\mathbf{m}_{h}, \mathbf{e}_{\perp}\right\rangle\right\|_{L^{2}(\omega)}^{2}:=\left\|\left\langle\mathbf{m}-\mathbf{m}_{h}, \mathbf{e}_{\perp}^{1}\right\rangle\right\|_{L^{2}(\omega)}^{2}+\left\|\left\langle\mathbf{m}-\mathbf{m}_{h}, \mathbf{e}_{\perp}^{2}\right\rangle\right\|_{L^{2}(\omega)}^{2}$. For two easy axes $\mathbf{e}=(0,0,1)$ and $\mathbf{e}=(1,2,3) / \sqrt{14}$, we compute approximations $\left(u_{h}, \mathbf{m}_{h}, \lambda_{h}\right)$ for $\left(\mathbf{P}_{h_{\mathcal{T}}, N}^{\mathrm{A}}\right)$, $\left(\mathbf{P}_{h_{\mathcal{T}}, N}^{\mathrm{B}}\right)$, and without stabilization. The corresponding numerical convergence results for $\left\|\nabla\left(u-u_{h}\right)\right\|_{L^{2}(\Omega)}$, $\left\|u-u_{h}\right\|_{L^{2}(\Omega)},\left\|\left\langle\mathbf{m}-\mathbf{m}_{h}, \mathbf{e}\right\rangle\right\|_{L^{2}(\omega)}$, and $\left\|\left\langle\mathbf{m}-\mathbf{m}_{h}, \mathbf{e}_{\perp}\right\rangle\right\|_{L^{2}(\omega)}$ versus the number of unknowns $N$ are plotted in Figure 13.

For a sequence of uniform meshes, we verify experimentally first order of convergence for error $=\left\|\nabla\left(u-u_{h}\right)\right\|_{L^{2}(\Omega)}+\left\|\left\langle\mathbf{m}-\mathbf{m}_{h}, \mathbf{e}_{\perp}\right\rangle\right\|_{L^{2}(\omega)}$ for both stabilized methods (slope $-1 / 3$, resp. $-2 / 3$ in Figure 13 corresponds to an experimental rate of convergence 1, resp. 2, owing to $N \propto h^{-3}$ in three dimensions). Our numerical experiments show a rate of convergence $\geq 2$ for $\left\|u-u_{h}\right\|_{L^{2}(\Omega)}$, for all meshes $(N \leq 44791)$. Without stabilization, no convergence for $\left\|\left\langle\mathbf{m}-\mathbf{m}_{h}, \mathbf{e}\right\rangle\right\|_{L^{2}(\omega)}$ can be obtained for both easy axes $\mathbf{e} \in \mathcal{S}^{2}$, and only poor convergence for $\left(\mathbf{P}_{h_{\mathcal{T}}, N}^{\mathrm{A}}\right)$ if $\mathbf{e}$ is aligned to the mesh $\mathcal{T}$. Only for stabilization $\operatorname{method}\left(\mathbf{P}_{h_{\mathcal{T}}, N}^{\mathrm{B}}\right)$, we 


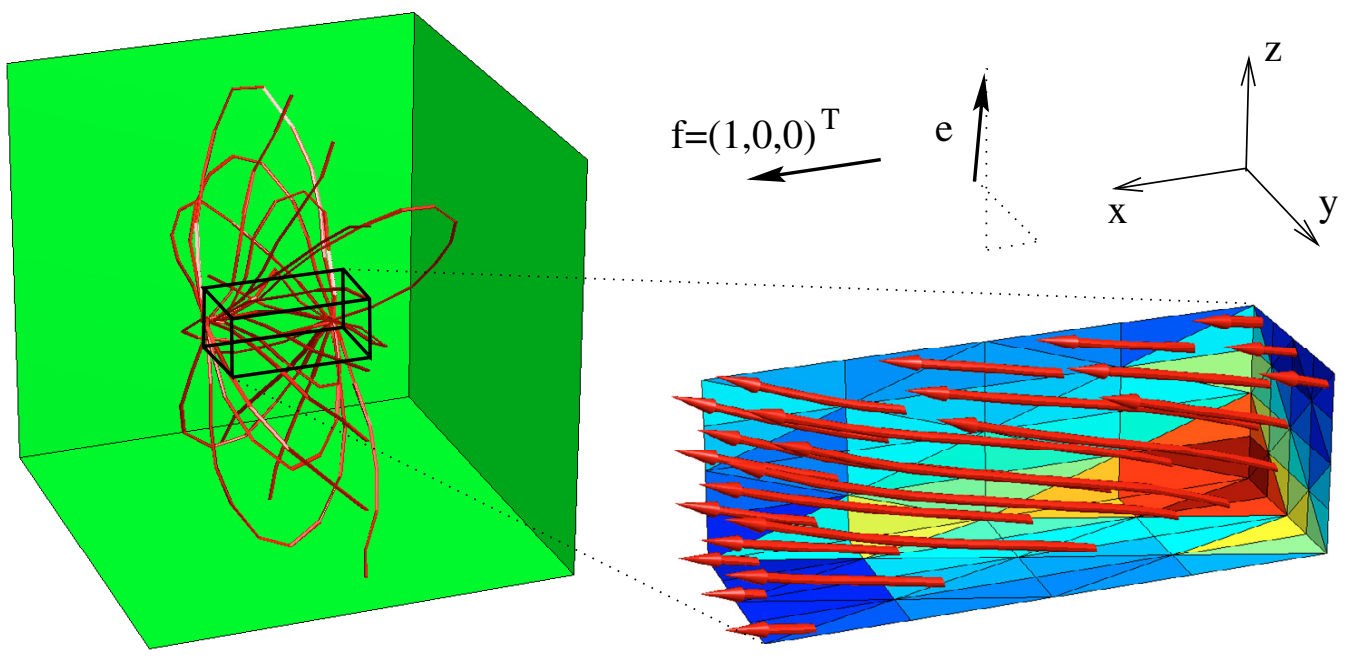

FiguRE 14. Streamlines of computed $\nabla u_{h}: \Omega \rightarrow \mathbb{R}^{3}$ (left) and approximated magnetization $\mathbf{m}_{h}: \omega \rightarrow \mathbb{R}^{3}$ (right) of Example 4.4, using $\left(\mathbf{P}_{h_{\mathcal{T}}, N}^{\mathrm{B}}\right)$.

find first order rate of convergence for both easy axes $\mathbf{e} \in \mathcal{S}^{2}$. However, we like to point out again that no theoretical justification for rates of convergence for the error $\left\|\left\langle\mathbf{m}-\mathbf{m}_{h}, \mathbf{e}\right\rangle\right\|_{L^{2}(\omega)}$ is available.

Example 4.4. Similar to Example 4.2, the approximate magnetization is computed in a 3D ferromagnetic rod by means of a stabilized method, using the data $\Omega=(0,3)^{3}, \omega=(1,2) \times(4 / 3,5 / 3)^{2}, \mathbf{f}=(1,0,0)^{T}$, $\mathbf{e}=(1,2,3) / \sqrt{14}$, and $\beta=0.1$ in $\left(\mathbf{P}_{\varepsilon, N}^{\mathrm{B}}\right)$. A plot of the solution potential $u_{h}: \Omega \rightarrow \mathbb{R}(h=0.2)$, as well as some magnified details of $\mathbf{m}_{h}: \omega \rightarrow \mathbb{R}^{3}$ are shown in Figure 14 .

The last experiments study nonstationary ferromagnetism governed by (1.18); see also (1.20)-(1.22) for the related incremental formulation, for every $\delta \geq 0$ : the discrete regularized, penalized, stabilized problem $\left(\tilde{\mathbf{P}}_{h_{\mathcal{T}}, N}^{\ell, j} \mid \mathcal{S}_{0}^{1}(\mathcal{T}) \times \mathcal{L}^{0}\left(\left.\mathcal{T}\right|_{\omega}\right)^{d} \times \mathcal{L}^{0}\left(\left.\mathcal{T}\right|_{\omega}\right)\right)$, shortly $\left(\tilde{\mathbf{P}}_{h_{\mathcal{T}}, N}^{\ell, j}\right)$, for $\ell \in\{\mathrm{A}, \mathrm{B}\}$, reads as follows, for $\delta>0$ :

For every $j=0, \ldots, T / \tau$, seek $\left(u_{h}^{j}, \mathbf{m}_{h}^{j}, \lambda_{h}^{j}\right) \in \mathcal{S}_{0}^{1}(\mathcal{T}) \times \mathcal{L}^{0}\left(\left.\mathcal{T}\right|_{\omega}\right)^{d} \times \mathcal{L}^{0}\left(\left.\mathcal{T}\right|_{\omega}\right)$ satisfying for all $\left(w_{h}, \boldsymbol{\mu}_{h}\right) \in \mathcal{S}_{0}^{1}(\mathcal{T}) \times \mathcal{L}^{0}\left(\left.\mathcal{T}\right|_{\omega}\right)^{d}$,

$$
\begin{aligned}
& \left(\nabla u_{h}^{j}, \nabla w_{h}\right)_{\Omega}-\left(\mathbf{m}_{h}^{j}, \nabla w_{h}\right)_{\omega}=0, \\
& \left(\nabla u_{h}^{j}, \boldsymbol{\mu}_{h}\right)_{\omega}+\left(D \phi^{* *}\left(\mathbf{m}_{h}^{j}\right), \boldsymbol{\mu}_{h}\right)_{\omega}+\left(\frac{\xi\left(\mathbf{m}_{h}^{j}\right)-\xi\left(\mathbf{m}_{h}^{j-1}\right)}{\sqrt{\left|\xi\left(\mathbf{m}_{h}^{j}\right)-\xi\left(\mathbf{m}_{h}^{j-1}\right)\right|^{2}+c_{\delta} \tau}} D \xi\left(\mathbf{m}_{h}^{j}\right), \boldsymbol{\mu}_{h}\right)_{\omega} \\
& +\left(\lambda_{h}^{j} \mathbf{m}_{h}^{j}, \boldsymbol{\mu}_{h}\right)_{\omega}+t_{\ell}\left(\left[\mathbf{m}_{h}^{j}\right],\left[\boldsymbol{\mu}_{h}\right]\right)=\left(\mathbf{f}^{j}, \boldsymbol{\mu}_{h}\right)_{\omega}, \\
& \lambda_{h}^{j}=\frac{1}{c_{\varepsilon} h_{\mathcal{T}}} \frac{\left(\left|\mathbf{m}_{h}^{j}\right|-1\right)_{+}}{\left|\mathbf{m}_{h}^{j}\right|} \quad \text { a.e. in } \omega,
\end{aligned}
$$

with $\mathbf{f}^{j}(\cdot)=\mathbf{f}(\cdot, j \tau)$, and $0<c_{\delta}=\mathcal{O}(1)$. Existence of solutions follows from a direct method variational argument.

Example 4.5. Let $\omega=\left(\frac{1}{4}, \frac{3}{4}\right)^{2}, \Omega=(0,1)^{2}$, with $\mathbf{e}=(0,1), \mathbf{f}(t)=(0,10 \sin (t)), T=3 \pi, \tau=T / 600$, and $\xi(\mathbf{m})=\langle\mathbf{m}, \mathbf{e}\rangle$. We fix $\left(h, c_{\delta}\right)=\left(\frac{1}{16}, \frac{1}{1000}\right)$; Figure 15 displays magnetization patterns at subsequent times close to the first switching time for the corresponding hysteresis loop Figure 1, for applied fields $\left\{\mathbf{f}_{h}^{j}\right\}_{j}$ vs. averaged magnetization $\frac{1}{|\omega|} \int_{\omega}\left\langle\mathbf{m}_{h}^{j}, \mathbf{e}\right\rangle \mathrm{d} \mathbf{x}$; compare also with [12], where corresponding results using a stable, 

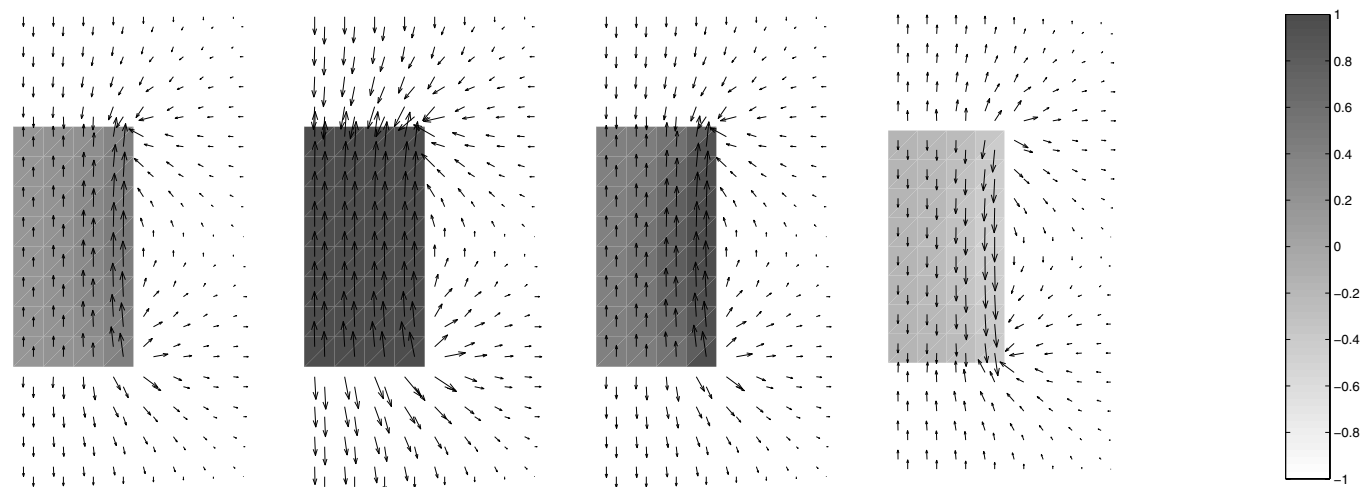

Figure 15. Example 4.5: computed magnetization (inside the magnet) and the self-induced magnetic field (outside the magnet) at four subsequent time steps A, B , C, D ( $c f$. Fig. 1), for $\left(h, c_{\delta}\right)=\left(\frac{1}{32}, \frac{1}{1000}\right)$.
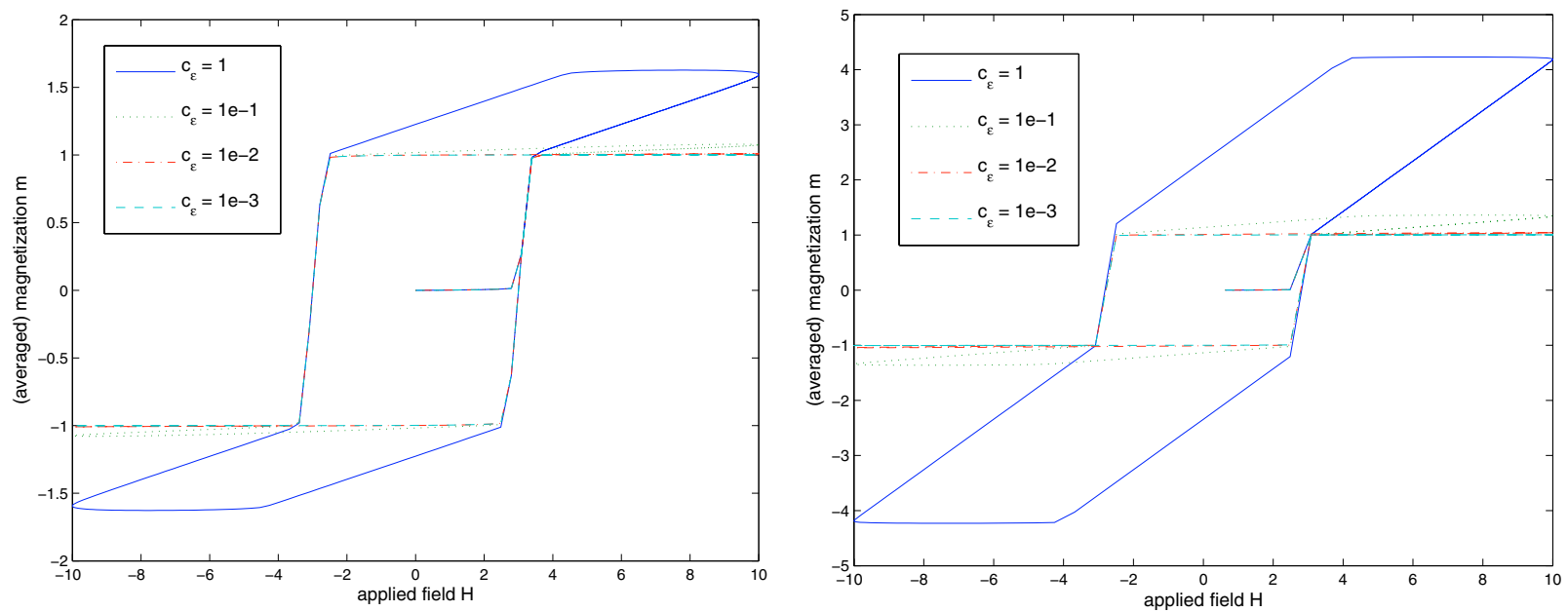

Figure 16. Hysteresis loops (Ex. 4.5 (left, $h=\frac{1}{16}$ ), Ex. 4.6 (right, $h=\frac{1}{9}$ )): applied field $\left\{\mathbf{f}_{h}^{j}\right\}_{j}$ vs. (averaged) magnetization $\frac{1}{|\omega|} \int_{\omega}\left\langle\mathbf{m}_{h}^{j}, \mathbf{e}\right\rangle \mathrm{d} \mathbf{x}\left(c_{\varepsilon}=1, \frac{1}{10}, \frac{1}{100}, \frac{1}{1000}\right)$.

nonconforming mixed discretization of (4.6)-(4.8) are obtained. Next, we study the influence of different values of parameters $(\varepsilon, \delta)$ : Figure 16 illustrates violation of convex side-constraint for too large values of $\varepsilon$; Figure 17 (right) evidences the effect of regularization parameter $\delta$ onto the shape of the hysteresis loop.

Dynamical behavior governed by (1.18) for the uniaxial case where $\omega$ is a 3D bounded ferromagnetic specimen is studied in the next example.

Example 4.6. Let $\Omega=(0,3)^{3}, \omega=(1,2) \times\left(\frac{4}{3}, \frac{5}{3}\right)^{2}$, e $=(0,0,1)$, and $\mathbf{f}(t)=(0,0,10 \sin (t)), T=3 \pi, T /$ $\tau=600$, and $\xi(\mathbf{m})=3\langle\mathbf{m}, \mathbf{e}\rangle$. Computational studies for $\left(h, c_{\delta}, c_{\varepsilon}\right)=\left(\frac{1}{9}, \frac{1}{1000}, \frac{1}{1000}\right)$ are performed. Figure 18 displays hysteresis loops for various $c_{\varepsilon}=\frac{1}{1000}, \ldots, 1$ and an applied field $\left\{\mathbf{f}_{h}^{j}\right\}_{j}$ vs. averaged magnetization $\frac{1}{|\omega|} \int_{\omega}\left\langle\mathbf{m}_{h}^{j}, \mathbf{e}\right\rangle \mathrm{d} \mathbf{x}$; compare with Example 4.5.

The last 2D example studies impact of a mixed ferromagnetic specimen onto hysteretic behavior. 

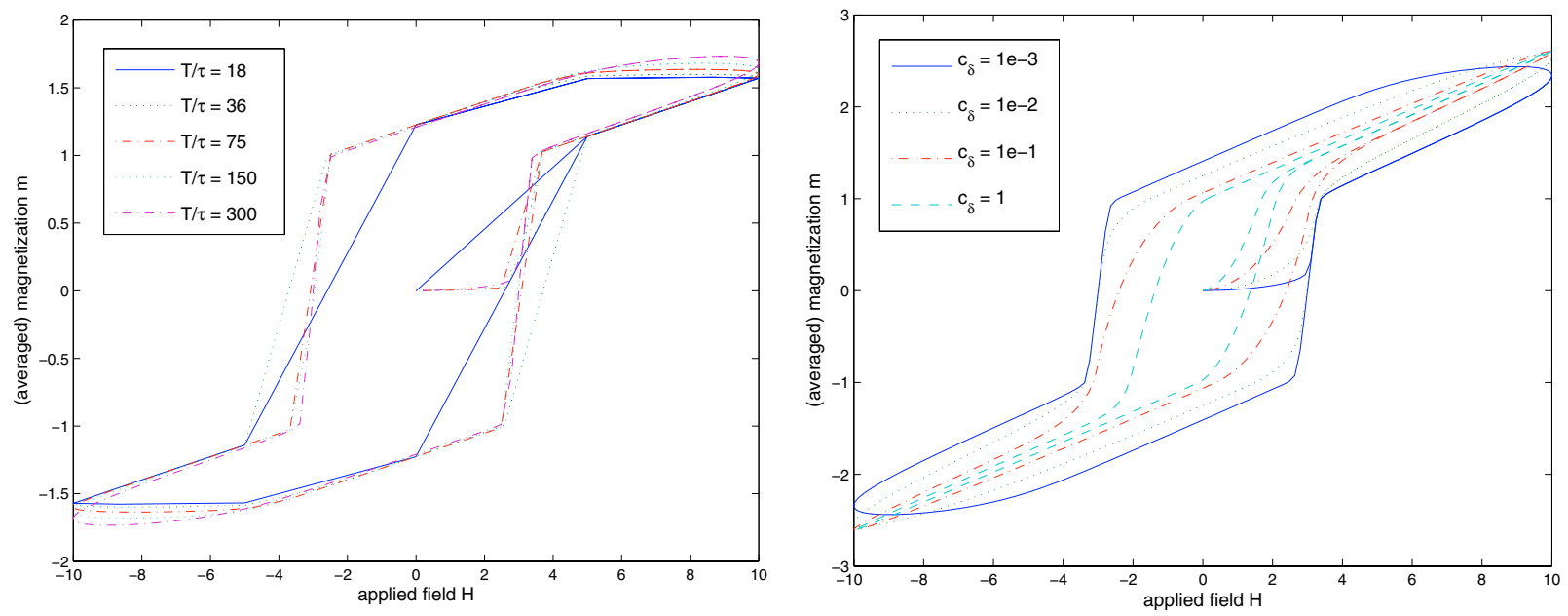

Figure 17. Hysteresis loops (Ex. $4.5\left(h=\frac{1}{16}, c_{\varepsilon}=1\right)$ ): applied field $\left\{\mathbf{f}_{h}^{j}\right\}_{j}$ vs. (averaged) magnetization $\frac{1}{|\omega|} \int_{\omega}\left\langle\mathbf{m}_{h}^{j}, \mathbf{e}\right\rangle \mathrm{d} \mathbf{x}\left(T / \tau=18,36,75,150,300\right.$ (left), $c_{\delta}=1, \frac{1}{10}, \frac{1}{100}, \frac{1}{1000}$ (right)).
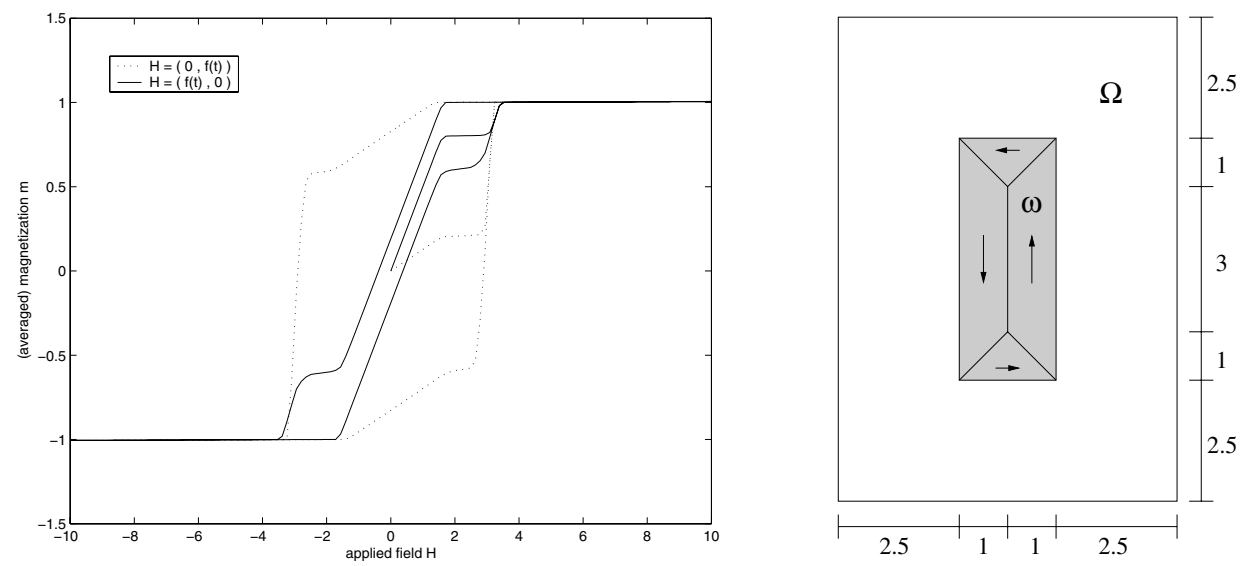

FiguRE 18. Example 4.7: computed magnetization (inside the magnet) and the self-induced magnetic field (outside the magnet) at six subsequent time steps $\left(\left(h, c_{\delta}\right)=\left(\frac{1}{32}, \frac{1}{1000}\right)\right)$.

Example 4.7. Let $\Omega=\left(-\frac{7}{2}, \frac{7}{2}\right) \times(-5,5), \omega=(-1,1) \times(-2,2), \mathbf{e}=\mathbf{e}(\mathbf{x})$, as depicted in Figure 18 (right), and $\mathbf{f}(t)=10 \sin (t) \overline{\mathbf{e}}$ with $\overline{\mathbf{e}}=(1,0)^{T}\left(\right.$ resp. $\left.\overline{\mathbf{e}}=(0,1)^{T}\right)$, for $T=3 \pi, T / \tau=600$, and $\xi(\mathbf{m})=3\langle\mathbf{m}, \mathbf{e}\rangle$. Computational studies for $\left(h, c_{\delta}, c_{\varepsilon}\right)=\left(\frac{1}{32}, \frac{1}{1000}, \frac{1}{1000}\right)$ are performed. Figure 18 displays hysteresis loops for applied fields $\overline{\mathbf{e}}^{T} \cdot\left\{\mathbf{f}_{h}^{j}\right\}_{j}$ vs. averaged magnetization $\frac{1}{|\omega|} \int_{\omega}\left\langle\mathbf{m}_{h}^{j}, \overline{\mathbf{e}}\right\rangle \mathrm{d} \mathbf{x} ;$ compare with Example 4.5.

Acknowledgements. Part of the results presented here are taken from the unpublished work [7]. This paper was partly written during S.A. Funken's stay at the "Forschungsinstitut für Mathematik" of ETH Zurich in April 2005. 


\section{REFERENCES}

[1] J. Alberty, C. Carstensen and S.A. Funken, Remarks around 50 lines of Matlab: finite element implementation. Numer. Algorithms 20 (1999) 117-137.

[2] W.F. Brown, Micromagnetics. Interscience, New York (1963).

[3] C. Carstensen and S. Funken, Adaptive coupling of penalised finite element methods and boundary element methods for relaxed micromagnetics. In preparation.

[4] C. Carstensen and D. Praetorius, Numerical analysis for a macroscopic model in micromagnetics. SIAM J. Numer. Anal. 42 (2005) 2633-2651, electronic.

[5] C. Carstensen and A. Prohl, Numerical analysis of relaxed micromagnetics by penalized finite elements. Numer. Math. 90 (2001) 65-99.

[6] A. De Simone, Energy minimizers for large ferromagnetic bodies. Arch. Rational Mech. Anal. 125 (1993) 99-143.

[7] S.A. Funken and A. Prohl, On stabilized finite element methods in relaxed micromagnetism. Preprint 99-18, University of Kiel (1999).

[8] A. Hubert and R. Schäfer, Magnetic Domains. Springer (1998).

[9] P. Keast, Moderate-degree tetrahedral quadrature formulas. Comput. Methods Appl. Mech. Engrg. 55 (1986) 339-348.

[10] M. Kružík, Maximum principle based algorithm for hysteresis in micromagnetics. Adv. Math. Sci. Appl. 13 (2003) $461-485$.

[11] M. Kružík and A. Prohl, Young measure approximation in micromagnetics. Numer. Math. 90 (2001) 291-307.

[12] M. Kružík and A. Prohl, Macroscopic modeling of magnetic hysteresis. Adv. Math. Sci. Appl. 14 (2004) 665-681.

[13] M. Kružík and A. Prohl, Recent developments in modeling, analysis and numerics of ferromagnetism. SIAM Rev. (accepted, $2005)$.

[14] M. Kružík and T. Roubíček, Microstructure evolution model in micromagnetics. Z. Angew. Math. Phys. 55 (2004) $159-182$.

[15] M. Kružík and T. Roubíček, Interactions between demagnetizing field and minor-loop development in bulk ferromagnets. J. Magn. Magn. Mater. 277 (2004) 192-200.

[16] P. Pedregal, Parametrized Measures and Variational Principles. Birkhäuser (1997).

[17] A. Prohl, Computational micromagnetism. Teubner (2001).

[18] R. Verfürth, A review of a posteriori error estimation and adaptive mesh-refinement techniques. Wiley-Teubner (1996).

To access this journal online: www.edpsciences.org 